\title{
Scoping assessment of building vibrations due to railway traffic
}

\author{
P. Galvín ${ }^{1}$, D. López Mendoza ${ }^{1}$, D.P. Connolly ${ }^{2}$, A. Romero ${ }^{1}$ \\ ${ }^{1}$ Escuela Técnica Superior de Ingeniería, Universidad de Sevilla, Camino \\ de los Descubrimientos s/n, 41092 Sevilla, Spain \\ ${ }^{2}$ Institute for High Speed Rail, Leeds University, LS2 9JT Leeds, UK
}

\section{Introduction}

Globally, the number of high speed railway (HSR) lines both operational and under construction is growing rapidly (e.g. HS2, UK). This has led to an increase in real estate located close to lines, and thus the number of properties effected by ground-borne vibrations (Connolly et al. (2015a)). Groundborne vibrations are vibrational waves generated within the track structure that propagate to nearby structures, causing shaking and in-door noise. These effects are undesirable and post-construction mitigation measures are expensive. Therefore vibration levels require prediction during early stages of planning/development, typically in the form of a desktop study.

International standard ISO2631 (2003a, $\mathrm{b})$ addresses these negative effects and evaluates the wholebody human exposure to vibration. In addition, ISO14837 (2005) is railway focused and describes the emission-propagation-immission mechanisms of waves from the train-track system (source) to the building (receiver). It provides a guide on the measurement of experimental data, vibration evaluation and mitigation. ISO14837 (2005) also outlines suggested numerical modelling approaches.

At locations of sensitive receptors (e.g. theatres) or where vibration has identified to likely to be a problem, comprehensive and detailed design models are often used. These are typically computationally expensive, and include three-dimensional (3D) models with full coupling between the train-track-soilstructure system (Galvín and Domínguez (2007); Galvín et al. (2010); Xia et al. (2010); Lopes et al. (2014); Auersch et al. (2014)). One alternative to 3D modelling is to use a two-and-a-half-dimensional (2.5D) approach (Lombaert et al. (2006); Lombaert and Degrande (2009); Auersch (2005); Sheng et al. (2006); Galvín et al. (2010); François et al. (2010); P. Alves Costa et al. (2012); Romero et al. (2015, 
2017); Jean et al. (2004)). These models assume the problem is continuous in the track direction and as such not well suited to modelling transition zones, etc.

At the earlier stage, when attempting to identify line sections where vibration is likely to cause problems in nearby buildings, simplified scoping models are often used. This is because they are faster running and allow engineers to assess long lengths of track quickly, in absence of detailed design information.

Empirical approaches to estimate soil and building vibrations due to a train passage have been proposed by the Federal Railroad Administration (FRA) and the Federal Transit Administration (FTA) of the U.S. Department of Transportation (Hanson et al. (2005, 2006)). The simplifications considered in these procedures have been verified by the numerical model presented by Verbraken et al. (2011). Alternatively, some scoping models have been recently proposed. Connolly et al. (2014a,b) presented a scoping tool, called Scoperail, to instantly compute vibrations due to train passages. A machine learning approach to obtain free-field vibrations was developed using numerically records for a wide range of train speeds and soil types. These soil vibrations were coupled with empirical factors in order to predict in-door noise and structural vibrations due to high speed trains. A hybrid model has been proposed by Triepaischajonsak and Thompson (2015), that combined a detailed vehicle-track model formulated in the time domain with a layered ground model operating in the frequency domain, based on the formulation outlined by Kausel and Roësset (1981). Then, forces acting on the ground were obtained from the train-track model and later used to calculate ground free-field vibrations. Kuo et al. (2016) developed a hybrid model where the source and propagation mechanisms were decoupled. The model combined experimental tests and numerical predictions considering the definitions proposed in Hanson et al. (2005, 2006). Kouroussis et al. (2017) developed a hybrid experimental-numerical model to predict vibrations from urban railway traffic. The level of vibration was calculated by combining the force density obtained from a numerical train-track model with the mobility function measured through an experimental approach.

Research has also been performed to investigate the propagation of free-field vibration into buildings. Auersch (2010) studied building responses using a simple soil-wall-floor model based on an empirical transfer fuction obtained from the characteristics of the structure. The soil was modelled using a spring and a viscous damper to evaluate the effects of soil-structure interaction. François et al. (2007) analysed building induced vibrations by employing simplified methods that ignore soil-structure interaction (SSI), but take into account the relative stiffness between the building and the soil. Later, Hussein et al. (2013) proposed a sub-modelling method to couple a 3D train-track-soil model with a 2D frame building. LópezMendoza et al. (2017) presented a scoping model, considering SSI, to predict vibrations in buildings 
induced by railway traffic from the ground-borne response spectra computed from either numerical or experimentally free field vibrations.

One challenge with the majority of vibration models, whether being used to predict free-field or structural vibration, is the unknown level of uncertainty in absolute levels. Therefore, in practise, it is common for vibration consultants to add a significant safety factor to predicted values. This is commonly of the order of $10 \mathrm{~dB}$, and means some areas where vibration will not be a problem, are then considered problematic. This can result in elevated project costs (e.g. unwarrented abatement measures).

Therefore this chapter presents a methodology to construct a conservative vibration scoping model, that does not require a safety factor to be applied to predicted values. The train-track-soil-building methodology considers soil stiffness, the combination of both the dynamic and static forces generated due to high speed train passage and SSI. It can be used to predict the vibration levels in the free-field and nearby buildings, using metrics compatible with international standards. These predictions need a fraction of the time typically required to analyse a complex SSI problem, and thus provides a practical tool to rapidly analyse the vibration response of several structures near railway lines.

The model uses the direct stiffness method to compute the soil's Green function (Kausel (2006); Kausel and Roësset (1981); Schevenels et al. (2009)), and a novel 2.5D finite element strategy for train-track interaction. The soil Green's function is modulated using a neural network (NN) to reduce the computation effort of track-soil interaction. This modulation factor combined with the new train-track approach results in a large reduction in computational time. The excitation of the building foundation corresponds with the soil response due to railway traffic. Structural vibration levels are computed using modal superposition, thus avoiding intensive computations.

In this chapter, the proposed scoping model is used to predict track receptance, free-field mobility, soil vibration and building response depending on track type, soil stiffness, building height and train speed. Also, the possibility of using the average shear wave velocity of a layered soil as defined in Eurocode 8 (1998) and denoted as $V_{s 30}$, to predict vibration levels, is investigated.

\section{Numerical modelling}

A wide variety of modelling approaches can be used to predict ground and building vibrations due to railway traffic. Typically, due to the large size of the modelling domain, the train-track-soil-building problem is divided into sub-models (Figure 1). These sub-models typically use simplified strategies, that can achieve similar accuracy, but in much reduced time. 


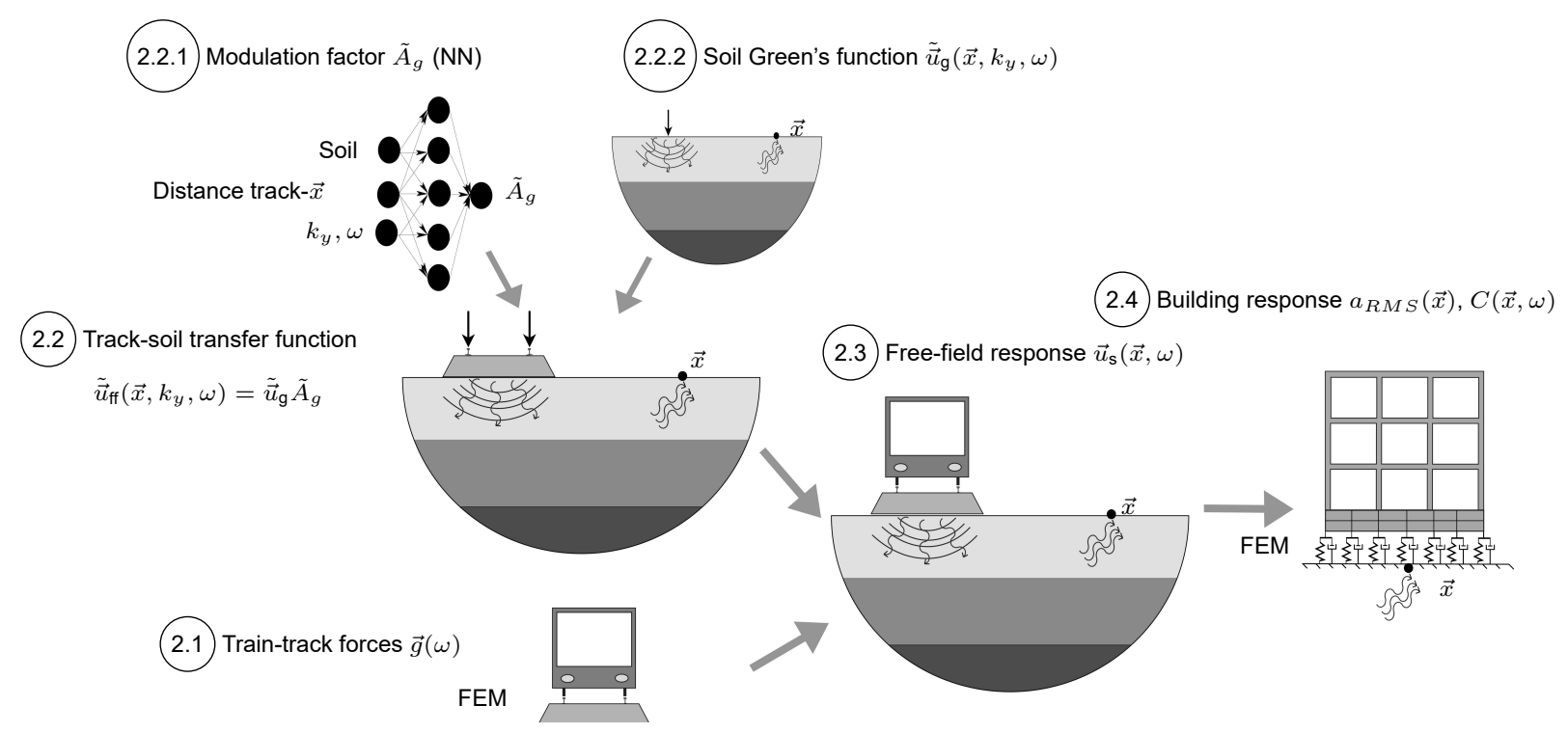

Figure 1. Scheme of the scoping model.

The proposed model (available as a MATLAB toolbox on the website http://personal.us.es/ pedrogalvin/scoping.en.html) assumes the train-track-soil interaction (source-propagation problem, Figure 1, step 2.3) can be decoupled from soil-structure interaction (immission problem, Figure 1, step 2.4). The simplified methodology presented by Galvín et al. (2018) is followed to calculate the free-field response (Figure1, step 2.3). The train-track-soil system is divided into two primary sub-models: a traintrack sub-model (Figure 1, step 2.1) and a track-soil sub-model (Figure 1, step 2.2). These sub-models are described below.

After obtaining the free-field response, it is used to compute the vibration within buildings located close to the line (Figure 1, step 2.4). To do so, the free-field response is the input for the soil-structure interaction model described in López-Mendoza et al. (2017).

To minimise the computational demand required, the following strategies are used:

- The train-track forces $\vec{g}$ are calculated using a simplified finite element (FEM) track model where the underlying soil is modelled using a spring-damper element that approximates the underlying soil response (step 2.1).

- The track-soil transfer function $\tilde{\vec{u}}_{\mathrm{ff}}$ (step 2.2) is approximated from the soil Green's function $\tilde{\vec{u}}_{\mathrm{g}}$ using a correction factor estimated using a neural network procedure.

- For the building response, the scoping model used a FEM approach based on a modal superposition analysis considering the SSI through a set of spring and damper elements at the foundation (step 2.4). 


\subsection{Track-soil forces}

\subsubsection{Track model}

The track-soil forces (Figure 1, step 2.1) are calculated using a simplified 2.5D FEM model (Figure 2). The model allows both linear hysteretic or viscous damping models for the constituents in the ballasted and slab track structure.

For the ballasted track model, the rails are represented using Euler-Bernoulli beams with a bending stiffness $E_{\mathrm{r}} I_{\mathrm{r}}$ and a mass $\rho_{\mathrm{r}} A_{\mathrm{r}}$ per unit length. The rail displacements are denoted as $u_{\mathrm{r} 1}\left(x_{1}, t\right)$ and $u_{\mathrm{r} 2}\left(x_{2}, t\right)$. The position of the rails is determined by the track gauge $w_{\mathrm{r}}$. The internal energy dissipation in the rail is modelled using a loss factor $\eta_{\mathrm{r}}$.

The rail pads are modelled as continuous spring-damper connections. The rail pad stiffness $k_{\mathrm{rp}}$ and damping coefficient $c_{\mathrm{rp}}$ are used to calculate the equivalent stiffness $\bar{k}_{\mathrm{rp}}=k_{\mathrm{rp}} / d_{\mathrm{sl}}$ and damping $\bar{c}_{\mathrm{rp}}=$ $c_{\mathrm{rp}} / d_{\mathrm{sl}}$, where $d_{\mathrm{sl}}$ is the sleeper spacing. Alternatively, a loss factor $\eta_{\mathrm{rp}}$ can be used to describe rail pad behaviour as, $\bar{k}_{\mathrm{rp}}=\bar{k}_{\mathrm{rp}}\left(1+i \eta_{\mathrm{rp}}\right)$.

The concrete sleepers are assumed to be rigid, so that the vertical sleeper displacements along the track are determined by the vertical displacement $u_{\mathbf{s l}}(x, t)$ and rotation $\theta_{\mathbf{s l}}(x, t)$ at the centre of gravity of the sleeper. The sleepers are modelled as a uniformly distributed mass $\bar{m}_{\mathbf{s l}}=m_{\mathbf{s l}} / d_{\mathrm{sl}}$, where $m_{\mathrm{sl}}$ is the mass of the sleeper. The rotational inertia of the sleeper is estimated as $\rho_{\mathrm{sl}} \bar{I}_{\mathrm{sl}}=\rho_{\mathrm{sl}} I_{\mathrm{sl}} / d_{\mathrm{sl}}$, where the inertia $I_{\mathrm{sl}}$ is calculated as $I_{\mathrm{sl}}=1 / 6 l_{\mathrm{sl}} h_{\mathrm{sl}} b_{\mathrm{sl}}\left(h_{\mathrm{sl}}^{2}+l_{\mathrm{sl}}^{2}\right)$, where $l_{\mathrm{sl}}, h_{\mathrm{sl}}$ and $b_{\mathrm{sl}}$ the sleeper length, height and width, respectively.

The ballast bed is represented by a set of distributed linear springs and dampers. The smeared ballast stiffness is computed from the vertical spring stiffness $k_{\mathrm{b}}$ per sleeper, as $\bar{k}_{\mathrm{b}}=k_{\mathrm{b}} / d_{\mathrm{sl}}$. The viscous damping in the ballast bed is accounted for using a complex impedance $\bar{k}_{\mathrm{b}}+i \omega \bar{c}_{\mathrm{b}}$. Alternatively, a loss factor $\eta_{\mathrm{b}}$ can be used to describe ballast behaviour as $\bar{k}_{\mathrm{b}}=\bar{k}_{\mathrm{b}}\left(1+i \eta_{\mathrm{b}}\right)$. The equivalent ballast mass $\bar{m}_{\mathrm{b}}$ is computed using the ballast mass $m_{\mathrm{b}}$ under each sleeper as $m_{\mathrm{b}} / d_{\mathbf{s l}}$. The ballast mass $m_{\mathrm{b}}$ is estimated from the height $h_{\mathrm{b}}$ of the ballast layer and lengths $l_{\mathrm{b} 1}=l_{\mathrm{s} 1}$ and $l_{\mathrm{b} 2}$ at the top and the bottom of the ballast layer, respectively, as $m_{\mathrm{b}}=0.5 \rho_{\mathrm{b}} h_{\mathrm{b}}\left(l_{\mathrm{b} 1}+l_{\mathrm{b} 2}\right) b_{\mathrm{s} \mathbf{l}}$.

The embankment is represented using a Euler-Bernoulli beam with a bending stiffness $E_{\mathrm{e}} I_{\mathrm{e}}$, a torsional rigidity $G_{\mathrm{e}} J_{\mathrm{e}}$, a loss factor $\eta_{\mathrm{e}}$, a rotational inertia $\rho_{\mathrm{e}} I_{p \mathrm{e}}$, and a mass $\rho_{\mathrm{e}} A_{\mathrm{e}}$ per unit length, where $E_{\mathrm{e}}, I_{\mathrm{e}}$, $G_{\mathrm{e}}, J_{\mathrm{e}}, I_{p \mathrm{e}}, \rho_{\mathrm{e}}$ and $A_{\mathrm{e}}$ are the Young's modulus, the bending moment of inertia, the shear modulus, the torsion constant, the polar moment of inertia, the density and the area, respectively. The embankment properties are approximated to be equal to the uppermost soil layer. 
A ballast mat can be simulated using spring-damper elements between the embankment and the ballast with equivalent stiffness and damping (or loss factor) $\bar{k}_{\mathrm{m}}$ and $\bar{c}_{\mathrm{m}}$ (or $\eta_{\mathrm{m}}$ ), respectively.
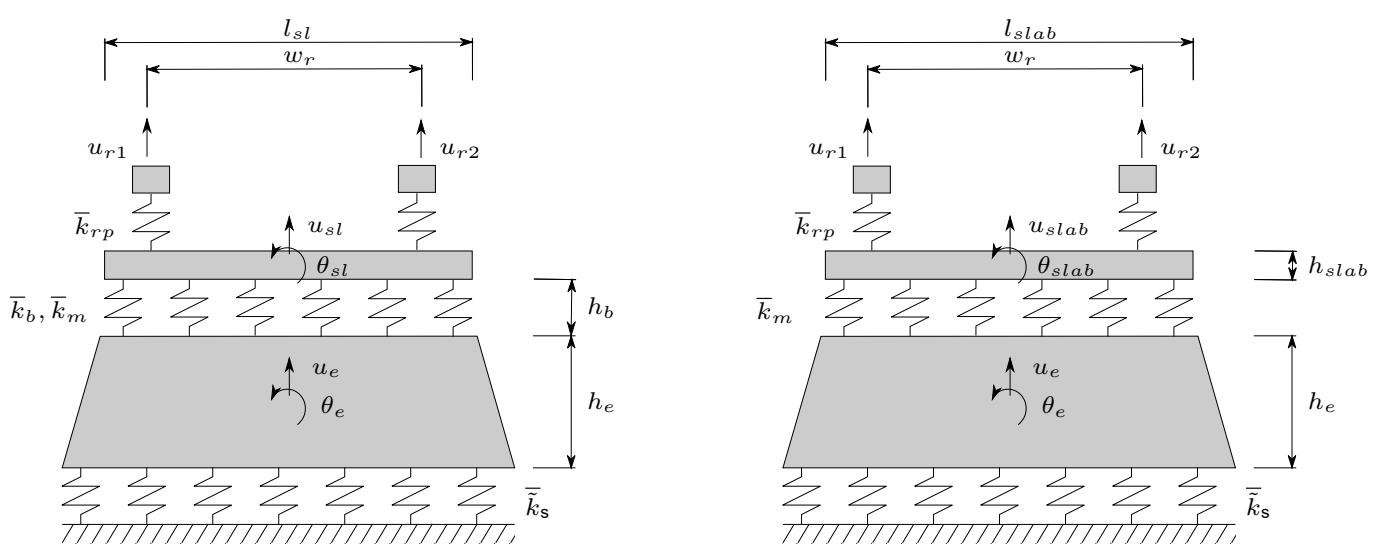

Figure 2. Cross section of (left) ballasted and (right) slab track models.

For the slab track model, the rails, rail pads and embankment are modelled in the same manner as the ballasted track model. The slab is a Euler-Bernoulli beam with a bending stiffness $E_{\text {slab }} I_{\text {slab }}$, a torsional rigidity $G_{\text {slab }} J_{\text {slab }}$, a rotational inertia $\rho_{\text {slab }} I_{p \text { slab }}$, a loss factor $\eta_{\text {slab }}$ and a mass per unit length $\rho_{\text {slab }} A_{\text {slab }}$, where $E_{\text {slab }}, I_{\text {slab }}, G_{\text {slab }}, J_{\text {slab }}, I_{p \text { slab }}, \rho_{\text {slab }}$ and $A_{\text {slab }}$ are the Young's modulus, the bending moment of inertia, the shear modulus, the torsion constant, the polar moment of inertia, the density and and the area, respectively.

The underlying soil is represented using a spring-damper element with stiffness $\overline{\tilde{k}}_{\mathbf{s}}\left(k_{y}, \omega\right)$. A tilde above a variable denotes its representation in the frequency-wavenumber domain. The equivalent stiffness and damping of the soil are estimated by the vertical soil response computed from the Green's function for a homogeneous or layered half-space due to a unit vertical load applied at a distance $w_{\mathrm{r}} / 2$ from the track axis. Note that because the spring-damper element does not consider the effect of a moving load, the model ignores the dynamic effects that may be induced when approaching critical velocity (e.g. Mezher et al. (2015)). The continuity of displacement is fulfilled between the soil and the track.

The 2.5D FEM formulation follows that outlined by Galvín et al. (2010):

$$
\left[-\omega^{2} \mathbf{M}_{\mathrm{bb}}+\mathbf{K}_{\mathrm{bb}}^{0}-i k_{y} \mathbf{K}_{\mathrm{bb}}^{1}-k_{y}^{2} \mathbf{K}_{\mathrm{bb}}^{2}+i k_{y}^{3} \mathbf{K}_{\mathrm{bb}}^{3}+k_{y}^{4} \mathbf{K}_{\mathrm{bb}}^{4}+\tilde{\mathbf{K}}_{\mathrm{bb}}^{\mathbf{s}}\left(k_{y}, \omega\right)\right] \tilde{\mathbf{u}}_{\mathrm{b}}\left(k_{y}, \omega\right)=\tilde{\mathbf{f}}_{\mathrm{b}}\left(k_{y}, \omega\right)
$$

where $\vec{K}_{\mathrm{bb}}^{0}, \vec{K}_{\mathrm{bb}}^{1}, \vec{K}_{\mathrm{bb}}^{2}, \vec{K}_{\mathrm{bb}}^{3}$ and $\vec{K}_{\mathrm{bb}}^{4}$ are the stiffness matrices, $\vec{M}_{\mathrm{bb}}$ is the mass matrix, $\tilde{\mathbf{f}}_{\mathrm{b}}\left(k_{y}, \omega\right)$ is the external load vector, and $\mathbf{K}_{\mathrm{bb}}^{\mathrm{s}}\left(k_{y}, \omega\right)$ represents the dynamic soil stiffness matrix. For simplicity, matrices $\vec{K}_{\mathrm{bb}}^{1}, \vec{K}_{\mathrm{bb}}^{2}$ and $\vec{K}_{\mathrm{bb}}^{3}$ are discarded so that the proposed model does not contain any volume or shell elements. The finite element matrices $\vec{M}_{\mathrm{bb}}, \vec{K}_{\mathrm{bb}}^{0}$ and $\vec{K}_{\mathrm{bb}}^{4}$ in Equation (1) are independent of wavenumber $k_{y}$ and frequency $\omega$, and are only assembled once. Equation (1) is now further elaborated by dividing 
the finite element degrees of freedom $\tilde{\mathbf{u}}_{\mathrm{b}}\left(k_{y}, \omega\right)$ into internal degrees of freedom $\tilde{\vec{u}}_{\mathrm{b}_{1}}\left(k_{y}, \omega\right)$ and degrees of freedom $\tilde{\vec{u}}_{\mathrm{b}_{2}}\left(k_{y}, \omega\right)$ for the soil-structure interface:

$$
\begin{aligned}
&\left(-\omega^{2}\left[\begin{array}{cc}
\vec{M}_{\mathrm{b}_{1} \mathrm{~b}_{1}} & \vec{M}_{\mathrm{b}_{1} \mathrm{~b}_{2}} \\
\vec{M}_{\mathrm{b}_{2} \mathrm{~b}_{1}} & \vec{M}_{\mathrm{b}_{2} \mathrm{~b}_{2}}
\end{array}\right]+\left[\begin{array}{cc}
\vec{K}_{\mathrm{b}_{1} \mathrm{~b}_{1}}^{0} & \vec{K}_{\mathrm{b}_{1} \mathrm{~b}_{2}}^{0} \\
\vec{K}_{\mathrm{b}_{2} \mathrm{~b}_{1}}^{0} & \vec{K}_{\mathrm{b}_{2} \mathrm{~b}_{2}}^{0}
\end{array}\right]+k_{y}^{4}\left[\begin{array}{cc}
\vec{K}_{\mathrm{b}_{1} \mathrm{~b}_{1}}^{4} & \vec{K}_{\mathrm{b}_{1} \mathrm{~b}_{2}}^{4} \\
\vec{K}_{\mathrm{b}_{2} \mathrm{~b}_{1}}^{4} & \vec{K}_{\mathrm{b}_{2} \mathrm{~b}_{2}}^{4}
\end{array}\right]+\right. \\
& {\left.\left[\begin{array}{cc}
0 & 0 \\
0 & \overrightarrow{\tilde{K}}_{\mathrm{b}_{2} \mathrm{~b}_{2}}^{\mathrm{s}}\left(k_{y}, \omega\right)
\end{array}\right]\right)\left[\begin{array}{l}
\tilde{\vec{u}}_{\mathrm{b}_{1}}\left(k_{y}, \omega\right) \\
\tilde{\vec{u}}_{\mathrm{b}_{2}}\left(k_{y}, \omega\right)
\end{array}\right]=\left[\begin{array}{c}
\tilde{\vec{f}}_{\mathrm{b}_{1}}\left(k_{y}, \omega\right) \\
\tilde{\vec{f}}_{\mathrm{b}_{2}}\left(k_{y}, \omega\right)
\end{array}\right] }
\end{aligned}
$$

The dynamic soil stiffness matrix $\tilde{\vec{K}}_{\mathrm{b}_{2} \mathrm{~b}_{2}}^{\mathrm{s}}\left(k_{y}, \omega\right)=\overline{\tilde{k}}_{\mathrm{s}}\left(k_{y}, \omega\right)$, which it is computed by means of the Green's function (Kausel and Roësset (1981)) (Figure 1 step 2.2.2).

The following section describes the evaluation of train-track interaction forces (Figure 1, step 2.1). Both quasi-static excitation and dynamic excitation due to random track unevenness are taken into account (Lombaert et al. (2014)). The dynamic contribution depends upon the rail displacements $\tilde{u}_{\mathrm{r}}\left(k_{y}, \omega\right)$ which are obtained from Equation (2).

\subsubsection{Train forces}

The train forces $\vec{g}(\omega)$ (Figure 1, step 2.1) are computed by the superposition of the dynamic $\vec{g}_{d}$ and quasi-static $\vec{g}_{q}$ excitations.

To compute the dynamic forces, a power spectral density (PSD) function is assumed for the simulation of random track unevenness (Lombaert et al. (2006)):

(3) $\quad \tilde{S}_{\mathrm{r} z z}\left(k_{y}\right)=\tilde{S}_{\mathrm{r} z z}\left(k_{y 0}\right)\left(\frac{k_{y}}{k_{y 0}}\right)^{-w}$

where $\tilde{S}_{\mathrm{r} z z}\left(k_{y 0}\right)$ is the reference value of the PSD at $k_{y 0}=1 \mathrm{rad} / \mathrm{m}$ and $w$ is the exponent that determines how the PSD function decreases with increasing wavenumber $k_{y}$. The coefficients $\tilde{S}_{\mathrm{r} z z}\left(k_{y 0}\right)$ and $w$ are obtained from standards.

The rail unevenness $\vec{u}_{w / r}(\omega)$ is evaluated as:

(4) $\quad \vec{u}_{w / r}(\omega)=\vec{T}(\omega, v) \frac{1}{v} \tilde{u}_{r z}\left(-\frac{\omega}{v}\right)$ 
where $\tilde{u}_{r z}\left(k_{y}\right)$ is the wavenumber transform of the rail unevenness $u_{r z}(y)$ and $\vec{T}(\omega)$ is a vector that collects the phase shift for each axle moving at a constant speed $v$, being:

$$
u_{r z}(y)=\sum_{m=1}^{n} \sqrt{2 \tilde{S}_{r z z}\left(k_{y m}\right) \Delta k_{y}} \cos \left(k_{y m} y-\theta_{m}\right)
$$

where $k_{y m}=m \Delta k_{y}$ is the wavenumber sampling, $\Delta k_{y}$ the wavenumber step, $n$ the size of the wavenumber sample and $\theta_{m}$ represents random phase angles uniformly distributed in the interval $[0,2 \pi]$.

The dynamic forces $\vec{g}_{d}(\omega)$ are computed from the track and vehicle compliances assuming a perfect contact between both (Lombaert et al. (2006)):

(6) $\quad \vec{u}_{c}(\omega)=\vec{u}_{r}(\omega)+\vec{u}_{w / r}(\omega)$

where $\vec{u}_{c}(\omega)$ contains the vehicle displacements at the train-track interface and both the rail displacements $\vec{u}_{r}(\omega)$ and the rail unevenness $\vec{u}_{w / r}(\omega)$ are evaluated at a fixed position in the moving frame of reference. The dynamic loads are computed as:

$$
\left[\vec{C}^{t}(\omega)+\vec{C}^{v}(\omega)\right] \vec{g}_{d}(\omega)=-\vec{u}_{w / r}(\omega)
$$

where $\vec{C}^{v}(\omega)$ is the vehicle compliance and $\vec{C}^{t}(\omega)$ is the track compliance.

The vehicle's unsprung mass $M_{u}$ is the only train mass considered when computing the vertical dynamic loads (Lombaert and Degrande (2009)). Then, the vehicle compliance is computed as $\vec{C}^{v}(\omega)=$ $\operatorname{diag}\left(-1 /\left(M_{u} \omega^{2}\right)\right)$. The vehicle compliance $C_{l k}^{v}$ represents the displacement at the contact point $k$ due to a unit load at the contact point $l$.

Additionally, track compliance $C_{l k}^{t}$ relates the track displacement at the position of axle $k$ due to a unit load at axle $l$. The track compliance is obtained from the rail impulse response $\tilde{u}_{\mathrm{r}}\left(k_{y}, \tilde{\omega}\right)$ (Equation (2)) using the following equation (Lombaert et al. (2006)):

$$
C_{l k}^{t}(\tilde{\omega})=\frac{1}{2 \pi} \int_{-\infty}^{+\infty} \tilde{u}_{\mathrm{r}}\left(k_{y}, \tilde{\omega}+k_{y} v\right) e^{-\mathrm{i} k_{y}\left(y_{l}-y_{k}\right)} \mathrm{d} k_{y}
$$

where $y_{l}$ and $y_{k}$ are the positions of $l$-th and $k$-th axles respectively. Also, the change of variables $\tilde{\omega}=\omega-k_{y} v$ is considered and $v$ is the train speed.

The quasi-static load is computed as:

(9) $\quad \vec{g}_{q}(\omega)=\sum_{k=1}^{n_{a}} w_{k} \exp \left(i \frac{\omega}{v} y_{k}\right)$ 
where $w_{k}$ and $y_{k}$ are the weight and the position of the $k$-th axle, while $n_{a}$ is the number of axles.

\subsection{Track-soil transfer function}

Many vibration prediction models consider track-soil interaction using comprehensive methodologies, which imply a high computational cost. In order to reduce the computational effort, the proposed model estimates the track-soil transfer function $\tilde{\vec{u}}_{\mathrm{ff}}\left(\vec{x}, k_{y}, \omega\right)$ (Figure 1, step 2.2) by combining the Green's functions $\tilde{\vec{u}}_{\mathrm{g}}\left(\vec{x}, k_{y}, \omega\right)$ (Kausel and Roësset (1981)) (Figure 1, step 2.2.2) for a homogeneous or layered soil with a correction factor $\tilde{A}_{g}$ obtained using a neural network (Figure 1, step 2.2.1). Note that the subindexes $\mathrm{ff}$ and $\mathrm{g}$ indicate free-field response and Green functions, respectively. The track-soil transfer function $\tilde{\vec{u}}_{\mathrm{ff}}\left(\vec{x}, k_{y}, \omega\right)$ represents the response at a point $\vec{x}=\{d, y, 0\}$ located at the soil surface due to an impulsive vertical load at the rail. Correction factor $\tilde{A}_{g}$ depends on the track type and the soil properties. It is evaluated for a point $\vec{x}$, a frequency $\omega$ and a wavenumber $k_{y}$. The track-soil transfer function at a point $\vec{x}$ can be obtained as:

$$
\tilde{\vec{u}}_{\mathrm{ff}}\left(\vec{x}, k_{y}, \omega\right)=\tilde{A}_{g}\left(d, k_{y}, \omega\right) \tilde{\vec{u}}_{\mathrm{g}}\left(\vec{x}, k_{y}, \omega\right)
$$

In order to estimate the correction factor, $\tilde{A}_{g}$, a multilayer perceptron (MLP) neural network architecture with a back-propagation training algorithm (Rumelhart and McClelland (1986)) is used. One, two and three hidden layers are tested. A NN framework with four layers (Figure 3: one input, two hidden and one output) is chosen to construct the proposed model.

The input layer (Figure 3) contains six inputs parameter: soil parameters $c_{s_{1}}, h_{1}, V_{s 30}$, the distance $d$ between the evaluated point $\vec{x}$ and the track, frequency $\omega$ and wavenumber, that is represented by the non-dimensional wavenumber $k_{d y}=k_{y} c_{s_{1}} / \omega . h_{1}$ and $c_{s_{1}}$ are the depth and the shear wave velocity of the upper layer respectively. Whereas, $V_{s 30}$ is the average shear wave velocity defined in Eurocode8 (1998):

$$
V_{s 30}=\frac{30}{\sum_{i}^{N_{s}} \frac{h_{i}}{c_{s_{i}}}}
$$

where $h_{i}$ is the thickness of the $i$ - th layer, $N_{s}$ the total number of layers in the top $30 \mathrm{~m}$ and $c_{s_{i}}$ the shear wave velocity of the $i$ - th layer. The shear wave velocity of the upper layer matches with the $V_{s 30}$ parameter $c_{s_{1}}=V_{s 30}$, with $h_{1}=30 \mathrm{~m}$ for an homogeneous soil.

The output layer has two parameters because the correction factor $\tilde{A}_{g}$ is a complex number. Therefore it is defined using its modulus $\left|\tilde{A}_{g}\right|$ (transformed to a logarithmic scale $K_{g}=20 \log _{10}\left|\tilde{A}_{g}\right|$ ) and argument $\arg \left(\tilde{A}_{g}\right)$ wrapped to $2 \pi$ rad. 


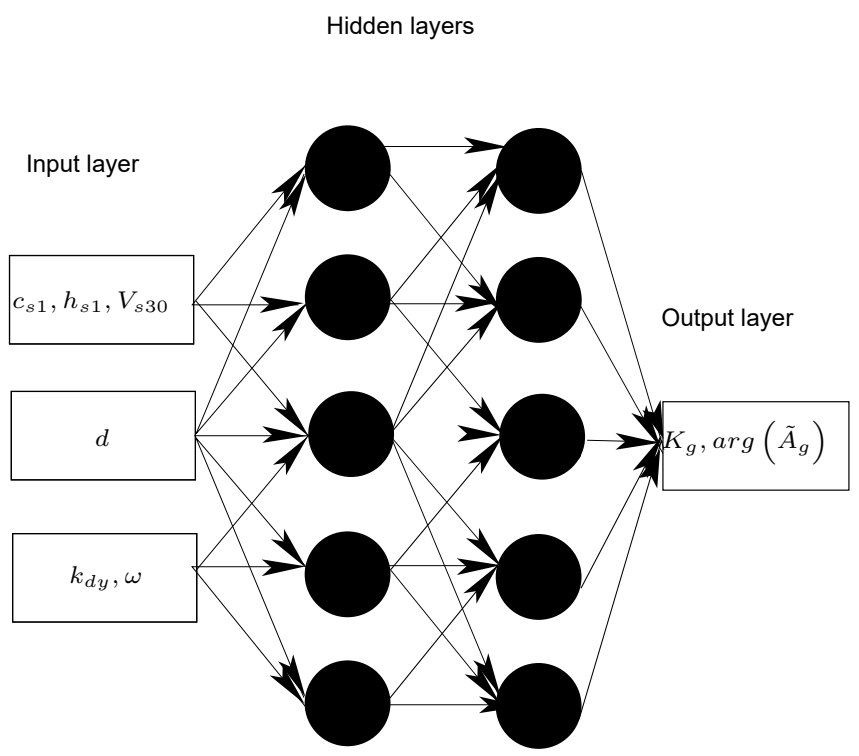

Figure 3. Neural network model schematic.

The aim of the NN procedure is to map the weighted inputs (e.g. distance) to outputs (i.e. vibration). First, weighted inputs are assumed and the resulting predicted outputs are compared against the known output targets to quantify the error. This error is fed back through the network using a back-propagation training algorithm. The input weightings are then modified and the process is repeated until convergence.

The NN approach is developed using the Matlab Neural Network Toolbox (Beale et al. (2017)). A tangent hyperbolic function is used as the activation function in the hidden layer due to its faster convergence compared to nonsymmetric functions (Cun et al. (1991)). The NN architecture is trained using the Levenberg-Marqurdt algorithm that has been be shown to be one of the faster methods for training NN (Hagan and Menhaj (1994)). Also, to evaluate the performance of the NN model and select the best framework, mean squared error $(M S E)$ and determination coefficient $\left(R^{2}\right)$ are used (Monjezi et al. (2010); Nourani and Fard (2012); Yurdakul and Akdas (2013)):

$$
M S E=\frac{1}{N_{n}} \sum_{i=1}^{N_{n}}\left(X_{i}-\hat{X}_{i}\right)^{2}
$$

(13) $R^{2}=1-\frac{\sum_{i=1}^{N_{n}}\left(X_{i}-\hat{X}_{i}\right)^{2}}{\sum_{i=1}^{N_{n}}\left(X_{i}-\operatorname{mean}(X)\right)^{2}}$ 
where $X_{i}$ and $\hat{X}_{i}$ are the output targets and predicted outputs, respectively, and $N_{n}$ is the size of the sample. Next expressions are used to build the output targets:

(14)

$$
\arg \left(\tilde{A}_{g}\right)=\arg \left(\tilde{\vec{u}}_{\mathrm{ff}}^{r}\right)-\arg \left(\tilde{\vec{u}}_{\mathrm{g}}\right)
$$

(15) $K_{g}=20 \log _{10}\left(\frac{\left|\tilde{\vec{u}}_{\mathrm{ff}}^{r}\right|}{\left|\tilde{\vec{u}}_{\mathrm{g}}\right|}\right)$

where $\tilde{\vec{u}}_{\mathrm{ff}}^{r}$ is the track-soil transfer function computed by using the reference model (Galvín et al. (2010); Galvín and Romero (2014)) (super-index $r$ ) and $\tilde{\vec{u}}_{\mathrm{g}}$ the Green's functions.

To train and evaluate the NN a large number of data output targets are used. Ballasted and slab tracks situated on top of an embankment are considered. Table 11 summarises the properties of track types (Figure 2). The material properties of the embankment are chosen equal to those of the top layer of the soil. A linear hysteretic damping model is used for all constituents of the ballasted and slab track structure.

A description of the NN database construction and performance is given in Galvín et al. (2018).

\subsection{Free-field response}

Once the track-soil transfer function $\tilde{\vec{u}}_{\mathrm{ff}}$ (Equation (10)), dynamic $\vec{g}_{d}$ (Equation (7)) and quasi-static $\vec{g}_{q}$ (Equation (9)) excitations are obtained, soil response $\vec{u}_{\mathbf{s}}(\vec{x}, \omega)$ due a train passage at speed $v$ is determined by following the $2.5 \mathrm{D}$ formulation in the wavenumber-frequency domain described in Lombaert et al. (2006). The free-field response $\vec{u}_{\mathrm{s}}(\vec{x}, \omega)$ is decomposed into its quasi-static $\vec{u}_{\mathrm{qs}}$ and dynamic $\vec{u}_{\mathrm{ds}}$ components as $\vec{u}_{\mathrm{s}}(\vec{x}, \omega)=\vec{u}_{\mathrm{qs}}(\vec{x}, \omega)+\vec{u}_{\mathrm{ds}}(\vec{x}, \omega)$. The quasi-static $u_{\mathrm{qsi}}$ and dynamic $u_{\mathrm{dsi}}$ contributions in the $i$-th direction at a point $\vec{x}$ is evaluated as:

$$
u_{\mathrm{qsi}}(\vec{x}, \omega)=\sum_{k=1}^{n_{a}} w_{k} \tilde{h}_{\mathrm{ffi}}\left(y-y_{k}, \omega, 0\right)
$$

$$
u_{\mathrm{dsi}}(\vec{x}, \omega)=\frac{1}{2 \pi} \sum_{k=1}^{n_{a}} \int_{-\infty}^{+\infty} \tilde{h}_{\mathrm{ffi}}\left(y-y_{k}, \omega, \tilde{\omega}\right) g_{\mathrm{d}_{k}}(\tilde{\omega}) \mathrm{d} \tilde{\omega}
$$

where $n_{a}$ is the number of axles and $w_{k}, y_{k}$ and $g_{\mathrm{d}_{k}}$ refer to weight, position and dynamic load of the $k$-th axle, respectively. A change of variables $\tilde{\omega}=\omega-k_{y} v$ is again considered and the relation used to 
Table 1. Ballasted and slab track properties.

\begin{tabular}{|c|c|c|}
\hline \multirow{3}{*}{ RAIL } & $\begin{array}{ll}\text { Bending stiffness } E_{\mathrm{r}} I_{\mathrm{r}} & \left.\mathrm{N} / \mathrm{m}^{2}\right]\end{array}$ & $6.18 \times 10^{6}$ \\
\hline & Mass per unit length $\rho_{\mathrm{r}} A_{\mathrm{r}}[\mathrm{kg} / \mathrm{m}]$ & 60.83 \\
\hline & Loss factor $\eta_{\mathrm{r}}$ & 0.05 \\
\hline \multirow{2}{*}{ RAIL PAD } & Equivalent stiffness $\bar{k}_{\mathrm{rp}}\left[\mathrm{N} / \mathrm{m}^{2}\right]$ & $150 \times 10^{6}$ \\
\hline & Loss factor $\eta_{\mathrm{rp}}$ & 0.25 \\
\hline \multirow{6}{*}{ SLEEPER } & Spacing $d_{\mathrm{sl}}[\mathrm{m}]$ & 0.6 \\
\hline & Length $l_{\mathrm{sl}}[\mathrm{m}]$ & 2.6 \\
\hline & Width $b_{\mathrm{sl}}[\mathrm{m}]$ & 0.35 \\
\hline & Height $h_{\mathrm{sl}}[\mathrm{m}]$ & 0.22 \\
\hline & Mass per sleeper $m_{\mathrm{sl}}[\mathrm{kg}]$ & 300 \\
\hline & Rotational inertia $\rho_{\mathrm{sl}} \bar{I}_{\mathrm{sl}}\left[\mathrm{kgm}^{2} / \mathrm{m}\right]$ & 567 \\
\hline \multirow{7}{*}{ BALLAST } & Length at the top $l_{\mathrm{b} 1}[\mathrm{~m}]$ & 2.6 \\
\hline & Length at the bottom $l_{\mathrm{b} 2}[\mathrm{~m}]$ & 2.87 \\
\hline & Width $b_{\mathrm{b}}[\mathrm{m}]$ & 0.35 \\
\hline & Height $h_{\mathrm{b}}[\mathrm{m}]$ & 0.3 \\
\hline & Equivalent mass $\bar{m}_{\mathrm{b}}[\mathrm{kg} / \mathrm{m}]$ & 796 \\
\hline & Vertical stiffness $k_{\mathrm{b}}[\mathrm{N} / \mathrm{m}]$ & $500 \times 10^{6}$ \\
\hline & Loss factor $\eta_{\mathrm{b}}$ & 1.0 \\
\hline \multirow{5}{*}{ SLAB } & Length $l_{\text {slab }}[\mathrm{m}]$ & 2.6 \\
\hline & Height $h_{\text {slab }}[\mathrm{m}]$ & 0.3 \\
\hline & Bending stiffness $E_{\text {slab }} I_{\text {slab }}\left[\mathrm{Nm}^{2}\right]$ & $117 \times 10^{6}$ \\
\hline & Mass per unit length $\rho_{\text {slab }} A_{\text {slab }}[\mathrm{kg} / \mathrm{m}]$ & 1950 \\
\hline & Loss factor $\eta_{\text {slab }}$ & 0.01 \\
\hline \multirow{3}{*}{ EMBANKMENT } & Length at the top $l_{\mathrm{e} 1}[\mathrm{~m}]$ & 3.5 \\
\hline & Length at the soil surface $l_{\mathrm{e} 2}[\mathrm{~m}]$ & 7 \\
\hline & Height $h_{\mathrm{e}}[\mathrm{m}]$ & 1.5 \\
\hline
\end{tabular}

express Equations (16) and (17) in compact forms is:

(18) $\tilde{h}_{\mathrm{ffi}}\left(y-y_{k}, \omega, \tilde{\omega}\right)=\frac{1}{v} \tilde{u}_{\mathrm{ffi}}\left(\vec{x}, \frac{\omega-\tilde{\omega}}{v}, \omega\right) \exp \left[-i \frac{\omega-\tilde{\omega}}{v}\left(y-y_{k}\right)\right]$

\subsection{Building response}

Next the foundation of structure is excited by a ground motion that corresponds with the free-field response $\vec{u}_{\mathrm{s}}$ calculated previously (Equations (16) and (17)). Then, the dynamic analysis of the building due to railway traffic is computed from the 3D FEM methodology presented in López-Mendoza et al. (2017). This methodology is based on a modal superposition analysis (Clough and Penzien (1975)), to assess an overall value of the response and the contribution of the modes to this response. However, the time domain history of the building response can not be obtained from the proposed model. Below this methodology is briefly recapitulated. 
Total building response $\vec{u}_{\mathrm{t}}$ (Figure 1, step 2.4) is defined as the superposition of the ground motion $\vec{u}_{\mathrm{s}}$ and structure deformation $\vec{u}_{\mathrm{b}}$ :

$$
\vec{u}_{\mathrm{t}}(t)=\vec{u}_{\mathrm{b}}(t)+\vec{r} \vec{u}_{\mathbf{s}}(t)
$$

where $\vec{u}_{\mathrm{S}}$ is the free-field response in the time domain and the influence matrix $\vec{r}$ defines the wave incidence on the structure. The structure deformation $\vec{u}_{\mathrm{b}}$ is obtained by modal superposition as:

(20) $\vec{u}_{\mathrm{b}}(t)=\sum_{i=1}^{N} \sum_{j=1}^{3} \phi_{i} \Gamma_{i}^{j} \xi_{i}^{j}(t)$

where $\phi_{i}$ is the $i$-th mode shape, $N$ the number of modes, and $\Gamma_{i}^{j}$ and $\xi_{i}^{j}$ the modal participation factor and the amplitude for the $i$-th mode at direction $j$, respectively.

The vibration level of the structure is evaluated using the overall root-mean-square (RMS) value of the acceleration (ISO2631 (2003a)):

(21) $a_{R M S}=\sqrt{\frac{1}{T} \int_{0}^{T} \ddot{\vec{u}}_{\mathrm{t}}^{2}(t) d t}$

where $T$ is the characteristic period defined by the standard DIN45672 (1995) where the structural response is assumed to be stationary. Substituting the building response (Equation (19)) and the structure deformation (Equation (20)) in Equation (21), and considering some assumptions in the methodology (López-Mendoza et al. (2017)), leads to the next simplified expression to estimate the overall RMS:

$$
a_{R M S}=\sqrt{H_{s}+H_{b}^{\prime}}
$$

where $\sqrt{H_{s}}$ and $\sqrt{H_{b}^{\prime}}$ are related to the contributions to the RMS value of the ground motion and the structural response respectively. $H_{s}$ is calculated as:

(23) $H_{s}=\frac{1}{M^{2}} \sum_{n=1}^{M} \sum_{j=1}^{3} r^{j^{2}}\left|\ddot{U}_{s}^{j}\left(f_{n}\right)\right|^{2}$

where $M=\frac{T}{\Delta t}, \ddot{U}_{s}^{j}\left(f_{n}\right)$ is the Discrete Fourier transform of $\ddot{u}_{s}^{j}\left(t_{n}\right), t_{n}$ is the time sampling and $f_{n}$ is the frequency sampling. On the other hand, $H_{b}^{\prime}$ is computed from superposition of the modes as 
$H_{b}^{\prime}=\sum_{i=1}^{N} H_{b i}^{\prime}$, where the participation $H_{b i}^{\prime}$ of the $i$-th mode is evaluated by:

(24) $H_{b i}^{\prime}=\phi_{i}^{2} \sum_{j=1}^{3}\left(\Gamma_{i}^{j} \Lambda_{i}^{j}\right)^{2}$

where $\Lambda_{i}^{j}$ is the ground-borne response spectra defined for the natural frequency $f_{i}$ at direction $j$. The ground-borne response spectra $\Lambda_{i}^{j}$ is obtained as:

(25) $\Lambda_{i}^{j}=\frac{1}{M} \sqrt{\sum_{n=1}^{M}\left|\ddot{\Xi}_{i}^{j}\left(f_{n}\right)\right|^{2}}$

where $\ddot{\Xi}_{i}^{j}\left(f_{n}\right)$ represents the Discrete Fourier transform of the amplitude $\ddot{\xi}_{i}^{j}\left(t_{n}\right)$ computed by solving the Duhamel's integral as (Clough and Penzien (1975)):

$$
\xi_{i}^{j}(t)=\frac{1}{f_{d i}} \int_{0}^{t}-\ddot{u}_{s}^{j} e^{-2 \pi \zeta_{i} f_{i}(t-\tau)} \sin \left(2 \pi f_{d i}(t-\tau)\right) d \tau
$$

where $\zeta_{i}$ is the damping ratio and $f_{d i}=f_{i} \sqrt{1-\zeta_{i}^{2}}$ the damped natural frequency.

The contribution of the $i$-th mode to the overall RMS value of the acceleration is estimated from Equations (22) and (24) as:

(27) $C_{i}=\sqrt{H_{b i}^{\prime}}$

Soil-structure interaction is considered by adding spring $k_{f}$ and damper $c_{f}$ elements to the foundation of the building model. Next correlations for shallow foundations (Auersch (2010)) are used:

(28) $k_{f}=3.4 G_{s} \sqrt{A_{f}}$

(29) $c_{f}=1.6 \sqrt{G_{s} \rho_{s} A_{f}}$

where $G_{s}$ and $\rho_{s}$ are the shear modulus and the mass density of the soil, respectively, and $A_{f}$ is the foundation area.

Finally, Table 2 summarises the main steps to compute track vibration generation and its propagation into buildings ((Figure 1). 
Table 2. Scoping model implementation.

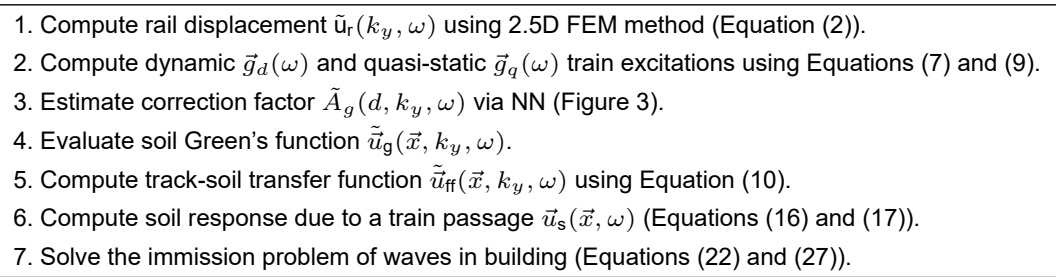

\section{Analysis}

An analysis of the effect of track, soil, building type and train speed on building vibration are now investigated. Two track types are considered. Track 1 is a classical ballasted track (Section 2.2 (Table 1)) supported by an embankment. Track 2 is a slab track (Figure 2) with identical rails, rail pads and embankment as Track 1 (Section 2.2 (Table 1)).

The structures are four, eight and twelve storey buildings with the same floor plan dimensions $12 \mathrm{~m} \times$ $12 \mathrm{~m}$ (Figure 4.a). It consists of eight concrete columns with $0.6 \mathrm{~m} \times 0.4 \mathrm{~m}$ section, four edge beams with $0.6 \mathrm{~m} \times 0.2 \mathrm{~m}$ section and two framed concrete walls with $2.4 \mathrm{~m} \times 0.15 \mathrm{~m}$ section. The floors are simply supported concrete slabs with a thickness of $0.2 \mathrm{~m}$. The floors consist of a two-dimensional frame with axial stiffness per unit length $E A=1.433 \times 10^{9} \mathrm{~N} / \mathrm{m}$, bending stiffness per unit length $E I=$ $9.935 \times 10^{6} \mathrm{Nm}$, and a mass per unit area of $m=172 \mathrm{~kg} / \mathrm{m}^{2}$. The structure is founded on a $1.0 \mathrm{~m}$ thick concrete slab. The concrete material has the following properties: Young's modulus $E=20 \times 10^{9} \mathrm{~N} / \mathrm{m}^{2}$, Poisson's ratio $\nu=0.2$, density $\rho=2400 \mathrm{~kg} / \mathrm{m}^{3}$ and Rayleigh damping is used, with $\zeta=0.05$ for all modes that contribute to the building response. The structure is discretised using two-node Euler-Bernoulli elements to represent columns and beams and four-node shell elements for floors and framed walls.

The midpoint of the foundation is located at distances $d=\{20,30,40,50\} \mathrm{m}$ from the track axis. The building response is evaluated using a single point response (SPR) excitation model, where the soil vibration is transmitted simultaneously to the whole foundation of the structure. It should be noted that as the building response is based upon the free-field response calculated using the scoping methodology, any errors are propagated through to the building calculation. The building responses at points $A$ and $B$ (Figure 4.(a)) located at the top floor are analysed.

Figure 5 presents the bending mode shapes of the floors computed without considering SSI for increasing edge (Figure 5. (a-c)) and framed wall (Figure 5. (d-f)) deformations.

The passage of a S-100 train travelling at $\{100,150,200\} \mathrm{km} / \mathrm{h}$ is studied. Table 3 shows the carriage length $L_{t}$, the distance between bogies $L_{b}$, the axle distance $L_{a}$, the total axle mass $M_{t}$ and the unsprung axle mass $M_{u}$ for all carriages. 


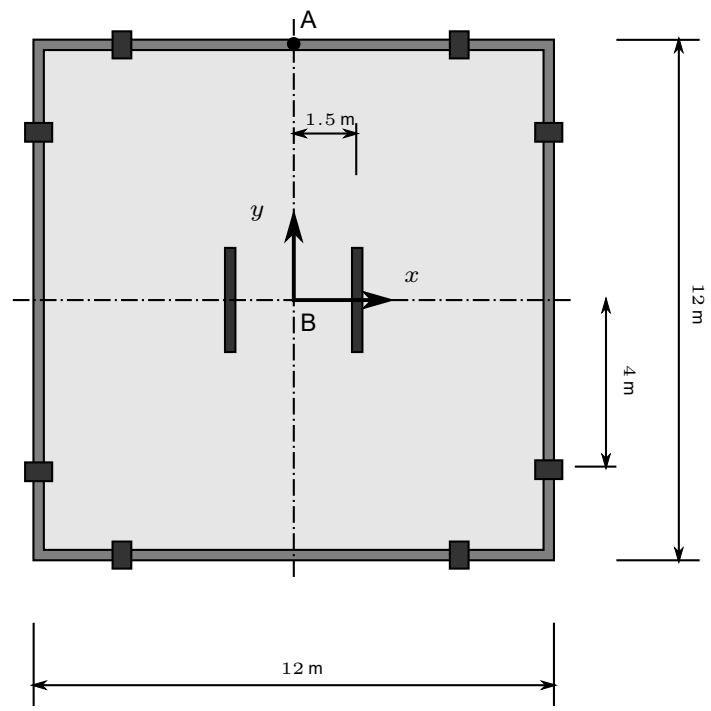

(a)

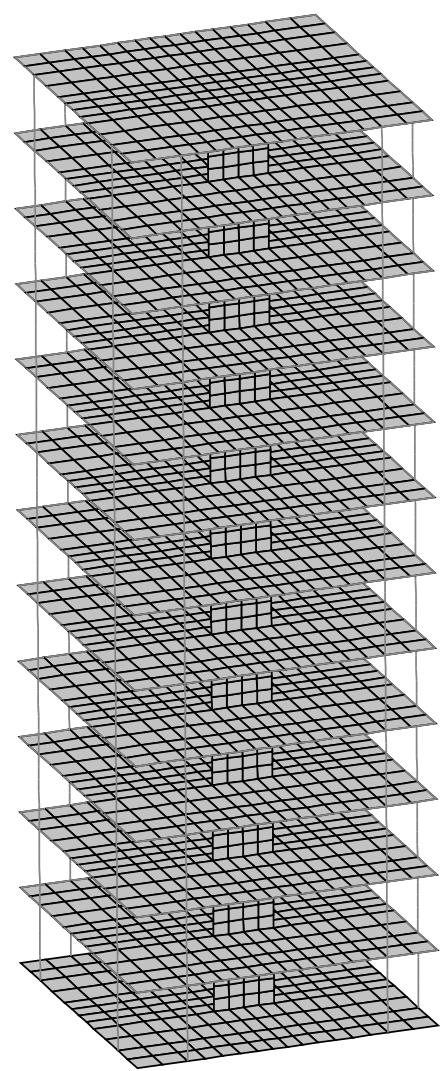

(b)

Figure 4. (a) Four, eight and twuelve-storey buildings plan geometry and (b) discretization of the twelve-storey building.

Table 3. Geometrical and mass characteristics of the S-100 train.

\begin{tabular}{lccccccc}
\hline & No. of carriages & No.of axles & $L_{t}[\mathrm{~m}]$ & $L_{b}[\mathrm{~m}]$ & $L_{a}[\mathrm{~m}]$ & $M_{t}[\mathrm{~kg}]$ & $M_{u}[\mathrm{~kg}]$ \\
\hline Traction cars & 2 & 4 & 22.15 & 14.00 & 3.00 & 17185 & 2048 \\
End carriages & 2 & 3 & 21.84 & 18.70 & 3.00 & 11523 & 2003 \\
Central carriages & 6 & 2 & 18.70 & 18.70 & 3.00 & 15523 & 2003 \\
\hline
\end{tabular}

Quasi-static and dynamic excitations are taken into account (Lombaert and Degrande (2009)) using the same track unevenness profile for all the studied cases. The dynamic contribution is expected to be dominant in the free-field response (Lombaert and Degrande (2009)) because the train speeds are below the critical velocity of the track system (P. Alves Costa et al. (2015)).

\subsection{Track type}

Next, the influence of the ballasted and slab tracks on the results is studied. Embankment properties are identical to the underlying soil modelled as a homogeneous elastic half-space with a shear wave velocity $c_{\mathrm{s}}=200 \mathrm{~m} / \mathrm{s}$, a dilatational wave velocity $c_{\mathrm{p}}=400 \mathrm{~m} / \mathrm{s}$ and density $\rho=1800 \mathrm{~kg} / \mathrm{m}^{3}$. The material damping ratio $\beta$ for both deviatoric and volumetric deformation has a value of 0.05 . 


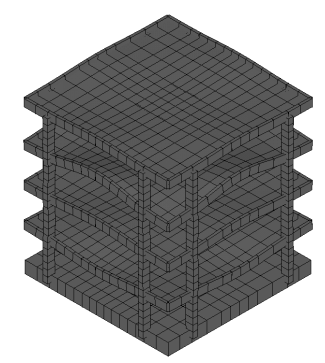

(a) Mode at $13.93 \mathrm{~Hz}$



(d) Mode at $50.63 \mathrm{~Hz}$

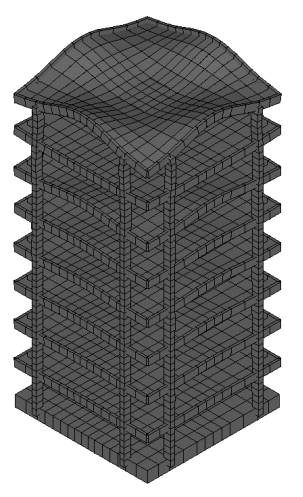

(b) Mode at $11.46 \mathrm{~Hz}$

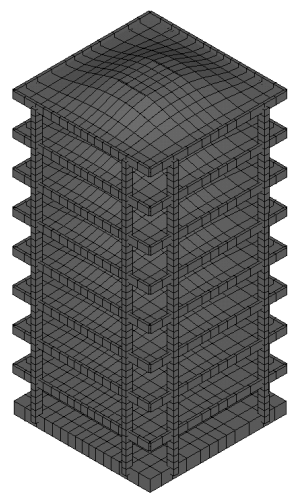

(e) Mode at $30.19 \mathrm{~Hz}$

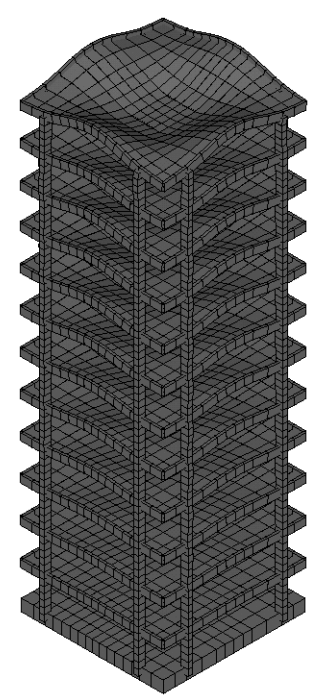

(c) Mode at $9.10 \mathrm{~Hz}$

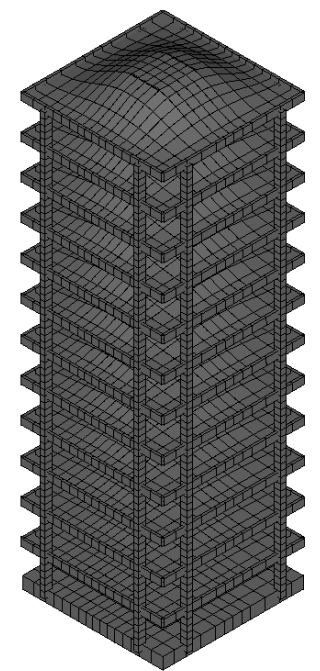

(f) Mode at $20.14 \mathrm{~Hz}$

Figure 5. Bending floor mode shapes of the ( $a$ and $d$ ) four-storey building, ( $b$ and e) eight-storey building and (c and f) twuelve-storey building.

Figure 6 shows the modulation of the dynamic loads and the free-field response due to the track type. The free-field response has been computed from the weighted acceleration with a time window of $1 \mathrm{~s}$ as prescribed by the ISO 2631 standard ISO2631 (2003a). It can be seen that the dynamic slab track loads are higher at frequencies above $40 \mathrm{~Hz}$. This is because slab track has a higher stiffness which also causes the free field response to increase at this frequency range. However, the soil response due to the train passage at the low and medium frequency ranges is attenuated by the slab track due to the effect of the free-field mobility (Figure 7). 


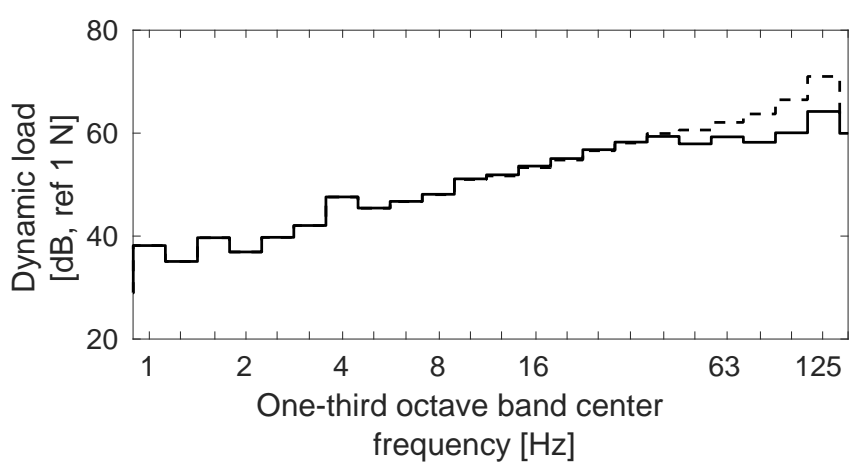

(a)

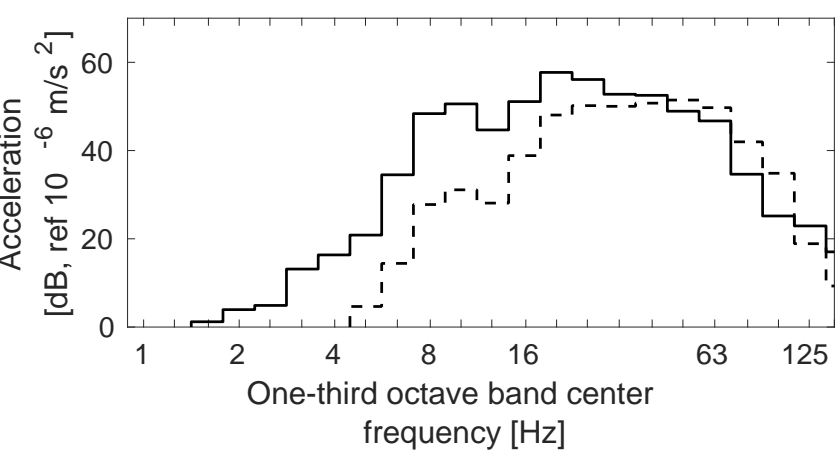

(b)

Figure 6. One-third octave band center frequency of the (a) dynamic load of an axle with unsprung mass $m_{s}=2048 \mathrm{~kg}$ and (b) the vertical weighted acceleration in the free-field at $20 \mathrm{~m}$ from the track due to a S-100 train passage, at $v=100 \mathrm{~km} / \mathrm{h}$ : (solid line) ballasted and (dashed line) slab tracks.

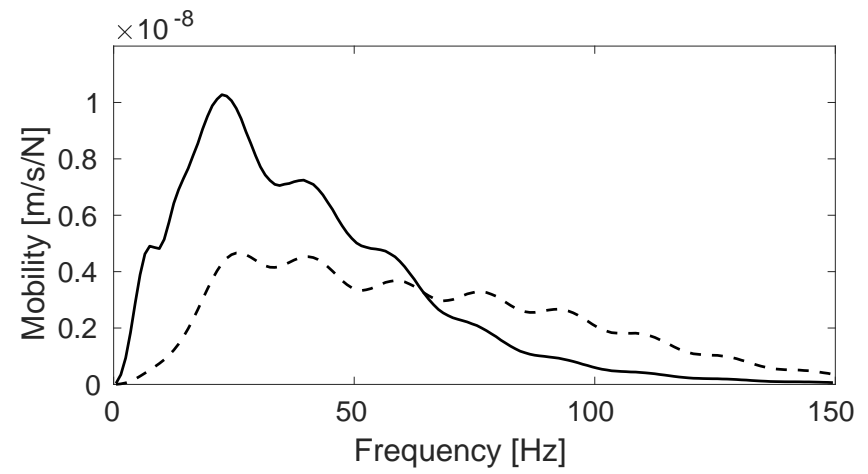

Figure 7. Free-field vertical mobility at $20 \mathrm{~m}$ from the axis track: (solid line) ballasted and (dashed line) slab tracks.

Figure 8 shows the maximum transient vibration value (MTVV) (ISO2631 (2003a)) of the free-field acceleration and the overall RMS value (Equation (21)) of the twelve storey building vibration level. The predicted vibration responses have been weighted according to ISO2631 (2003a) to obtain these values. Note that building response is computed considering the weighted ground motion $\ddot{\vec{u}}_{\mathrm{s}}$ (Equations (23) and (25)). Results for the ballasted track present the highest vibrations. As expected, free-field and building responses are attenuated with increasing distance from the track axis. The building vibration level is higher than the free-field response being this amplification more important for the ballasted track in this case.

\subsection{Soil properties}

The influence of the soil stiffness on the scoping model predictions is studied using three homogeneous soils (Eurocode8 (1998), Table 4): soft, medium and stiff soils. Their properties are shown in Table 5.

Figure 9 shows the influence of soil stiffness on the rail receptance. The response decreases with increasing soil stiffness. Furthermore, rail displacement is smoother and the dominant frequency 


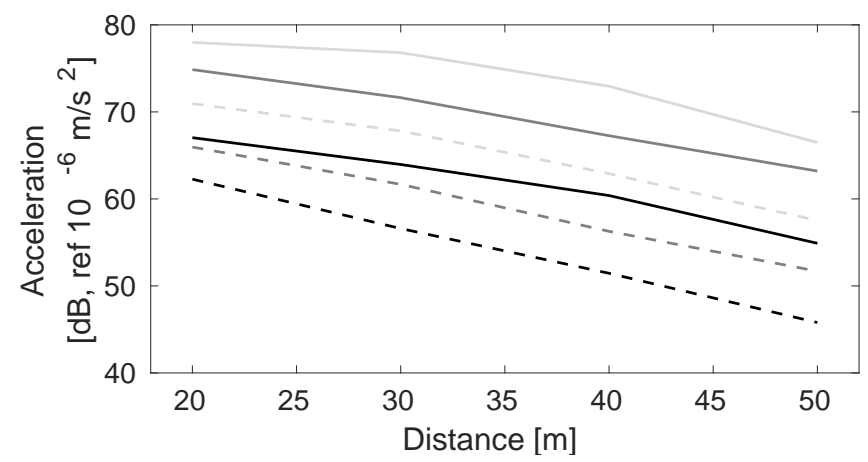

Figure 8. (Black line) MTVV at the free-field and overall RMS value of the weighted acceleration at the top floor of the observation points (dark grey line) $A$ and (light grey line) $B$, depending on the distances from the track due to a S-100 train passage at $v=100 \mathrm{~km} / \mathrm{h}$ considering (solid line) ballasted and (dashed line) slab tracks.

Table 4. Soil types based on Eurocode 8.

\begin{tabular}{c|cc}
\hline & Description & $V_{s 30}[\mathrm{~m} / \mathrm{s}]$ \\
\hline A & Rock outcrop & $>800$ \\
\hline B & Very dense sand or gravel, or very stiff clay & $360-800$ \\
\hline C & Dense to medium-dense sand or gravel, or stiff clay & $180-360$ \\
\hline D & Loose-to-medium sand or gravel & $<180$ \\
\hline
\end{tabular}

Table 5. Homogeneous soil properties.

\begin{tabular}{l|cccc}
\hline & $c_{p}[\mathrm{~m} / \mathrm{s}]$ & $c_{s}[\mathrm{~m} / \mathrm{s}]$ & $\xi[-]$ & $\rho\left[\mathrm{kg} / \mathrm{m}^{3}\right]$ \\
\hline Soft soil & 345.2 & 172.6 & 0.05 & 1800 \\
\hline Medium soil & 669.8 & 334.9 & 0.05 & 1800 \\
\hline Stiff soil & 993.6 & 496.8 & 0.05 & 1800 \\
\hline
\end{tabular}

increases as soil stiffness increases. This is because the differences between the stiffness of the embankment and the ballast are lower for the medium and stiff soils compared to the soft soil.



Figure 9. The displacement of the rail for (black line) soft, (dark grey line) medium and (light grey line) stiff soils (Table 5), considering the ballasted track. 
Regarding the dependence on soil stiffness of the track-soil transfer function estimations, free-field mobility at a distance of $20 \mathrm{~m}$ from the axis track is presented in Figure 10. Again, the response decreases and the dominant frequency increases with soil stiffness.

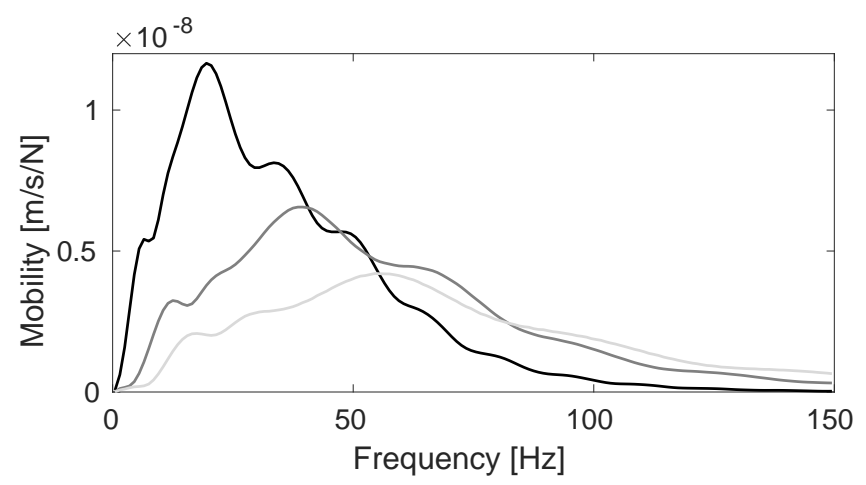

Figure 10. Free-field vertical mobility at $20 \mathrm{~m}$ from the ballasted track for (black line) soft, (dark grey line) medium and (light grey line) stiff soils (Table 5).

The effect of soil stiffness in the ground-borne response spectra and building vibrations due to the train passage is presented in Figure 11. The ground-borne response spectra $\Lambda_{i}^{j}\left(f_{i}\right)$ is computed from the weighted ground motion $\ddot{\vec{u}}_{\mathrm{s}}$ using Equation (25), considering a damping ratio $\zeta=0.05$ for all mode shapes. The contribution to the overall RMS value of the response of the building mode shapes, within a frequency band centred at $\Omega_{j}$, is computed as:

$$
C_{j}\left(\Omega_{j}\right)=\sum_{i} \sqrt{C_{i}^{2}\left(f_{i}\right)} \quad \forall f_{i} \in\left[\Omega_{j 0}, \Omega_{j 1}\right]
$$

where $\Omega_{j 0}$ and $\Omega_{j 1}$ are the limits of the one-third octave band center frequency $\Omega_{j}$, and $C_{i}$ is calculated from Equation (27).

According to the previous results (Figure 10), the frequency content of the ground-borne response spectra (Figure 11.a) shows that dominant frequencies due to the dynamic excitation vary from 10 to $50 \mathrm{~Hz}$ for the soft soil, to 20 and $60 \mathrm{~Hz}$ for the medium and stiff soils. Building response is most dominant distributed between frequencies from $8 \mathrm{~Hz}$ to $80 \mathrm{~Hz}$. The observation point $B$ presents the highest response (Figure 11.c). This is because point $B$ is located at the part of the slab supported by the frame walls, where the bending stiffness of the floor is higher than at the edge (point $A$ ). The response at point $A$ is concentrated at approximately $9 \mathrm{~Hz}$ corresponding to the mode with highest displacements on the edge (Figure 5. (c)), whereas maximum level of vibration at point $B$ is found at approximately $20 \mathrm{~Hz}$ matching the mode associated with highest framed wall deformation. According to the differences in the ground-borne response spectra (Figure 11.a). at the natural frequencies (9 and $20 \mathrm{~Hz}$ ) of the building (Figure 5. (c and f)), building response is higher for the soft soil (Figure 11.b and c). 


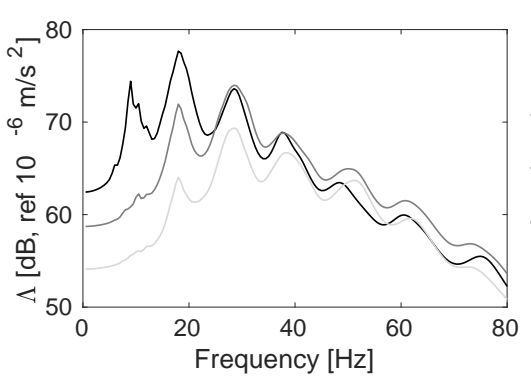

(a)

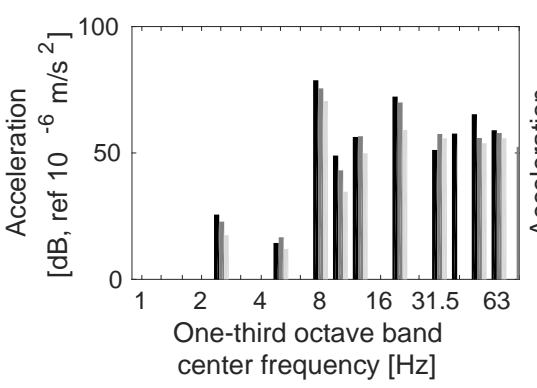

(b)

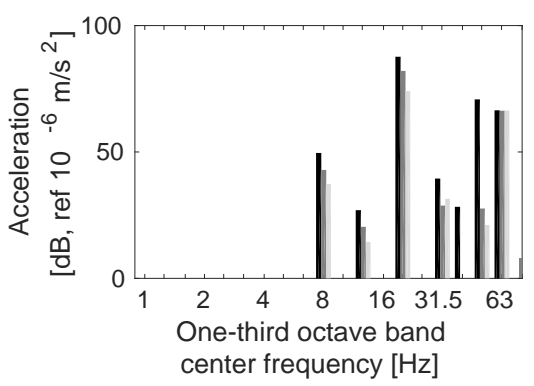

(c)

Figure 11. (a) Ground-borne response spectra $\Lambda_{i}^{j}\left(f_{i}\right)$ and (b and c) contribution of the modes to the overall RMS value of the vertical weighted acceleration at the top floor of the observation points (b) $A$ and (c) $B$, due to a S-100 train passage at $v=100 \mathrm{~km} / \mathrm{h}$ at $20 \mathrm{~m}$ from the ballasted track for (black line) soft, (dark grey line) medium and (light grey line) stiff soils (Table 5).

\subsection{Building height}

The effect of building height on building response computed from the scoping model is now studied considering the passage of a S-100 train travelling at $100 \mathrm{~km} / \mathrm{h}$ on the ballasted track on an embankment (Table 1) supported by a homogeneous soil with $c_{s}=200 \mathrm{~m} / \mathrm{s}$ (Section 3.1). Figure 12 shows the contribution of the modes to the overall RMS value of the vertical vibration. Dominant frequencies for the four, eight and twelve-storey buildings are found at approximately $12 \mathrm{~Hz}, 10 \mathrm{~Hz}$ and $8 \mathrm{~Hz}$ for observation point $\mathrm{A}$, and $50 \mathrm{~Hz}, 30 \mathrm{~Hz}$ and $20 \mathrm{~Hz}$ for observation point $\mathrm{B}$ respectively. Thus, the natural frequencies decrease as the building height increases. Also, there are slight differences in the response magnitude of the dominant modes.

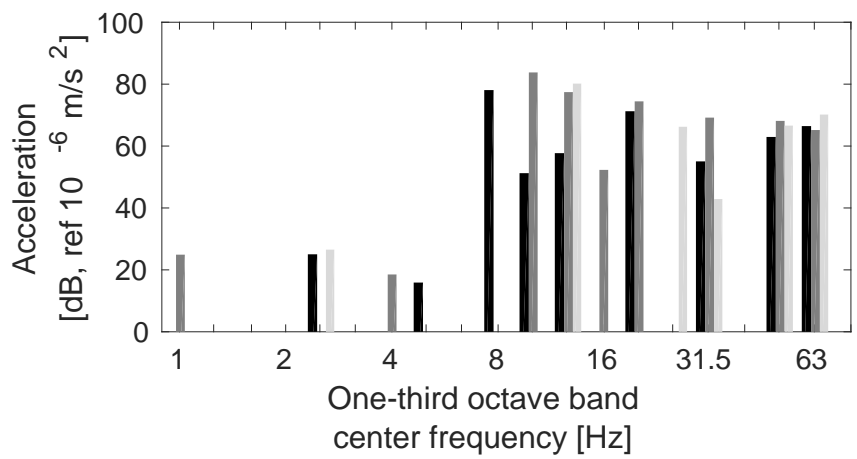

(a)

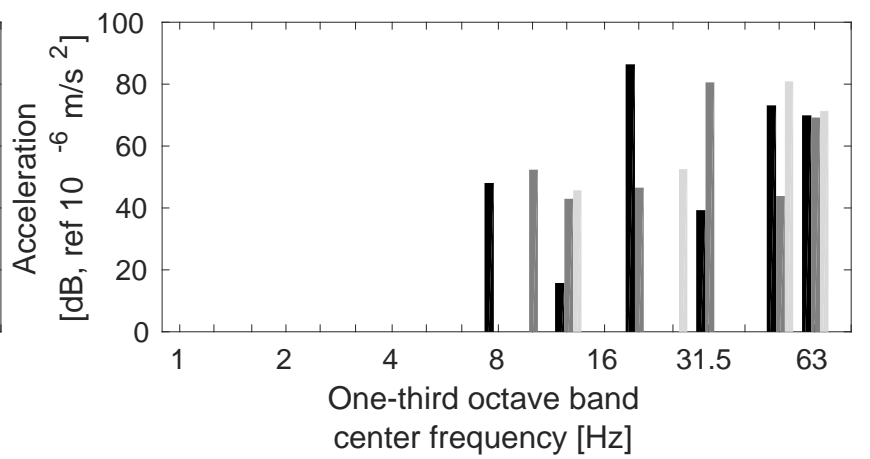

(b)

Figure 12. Contribution of modes to the overall RMS value of the vertical weighted acceleration of observation points (a) $A$ and (b) $B$ at the top floor, due to a S-100 train passage at $v=100 \mathrm{~km} / \mathrm{h}$ at $20 \mathrm{~m}$ from the ballasted track for the (light grey bars) four-storey building, (dark grey bars) eight-storey building and (black bars) twelve-storey building.

Figure 13 presents the influence of building height on the overall RMS value of response. The results are shown for different storey levels. The response increases with storey level, however, this correlation is not observed at observation point $A$ for the four-storey building (Figure 13.(a)). This can be explained 
from the dominant response at observation point $A$ for the four-storey building (Figure 12.(a)) where the natural frequency $(13.93 \mathrm{~Hz}$ ) of a bending mode (Figure 5. (a)) experiences larger amplitudes at the middle floors of the building than at the top floor. Differences in the vibration level on the top floor, depending on the building height, are within a narrow range of $5 \mathrm{~dB}$.

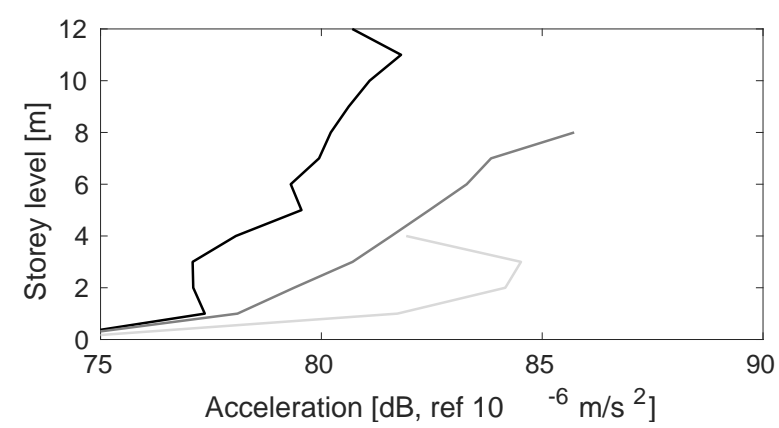

(a)

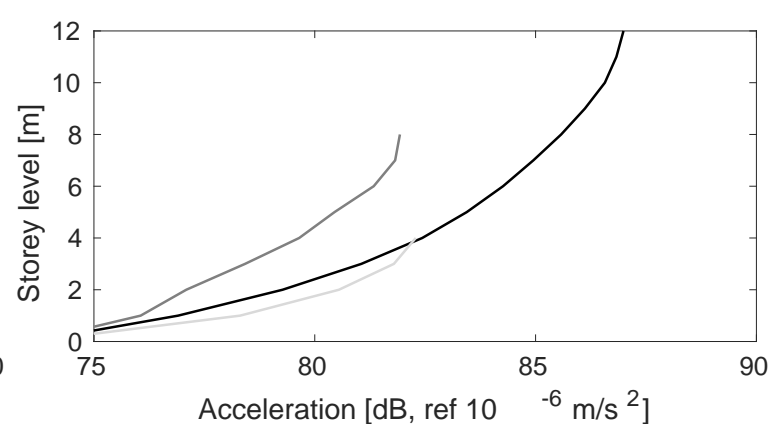

(b)

Figure 13. Overall RMS value of the weighted acceleration at the observation points (a) $A$ and (b) $B$ depending on the storey level, due to a S-100 train passage at $v=100 \mathrm{~km} / \mathrm{h}$ at $20 \mathrm{~m}$ from the ballasted track for the (light grey line) four-storey building, (dark grey line) eight-storey building and (black line) twelve-storey building.

\subsection{Train speed}

Next, the scoping model is used to assess the effect of train speed on railway vibrations. The passage of a S-100 train travelling at $\{100,150,200\} \mathrm{km} / \mathrm{h}$ is simulated. The ballasted track on a top of an embankment (Table 1) and a homogeneous medium with $c_{s}=200 \mathrm{~m} / \mathrm{s}$ are considered again.

Figure 14 presents the influence of the train speed on the free-field predictions of the proposed model. The quasi-static contribution is observed in the frequency content at approximately the axle passing frequency $f_{a}=v / L_{a}=\{9.26,13.9,18.52\} \mathrm{Hz}$. The dominant frequency due to the dynamic excitation remains in the range $20-40 \mathrm{~Hz}$ for the different train speeds. Both quasi-static and dynamic contributions increase with train speed, however it is more noticeable for the quasi-static case.

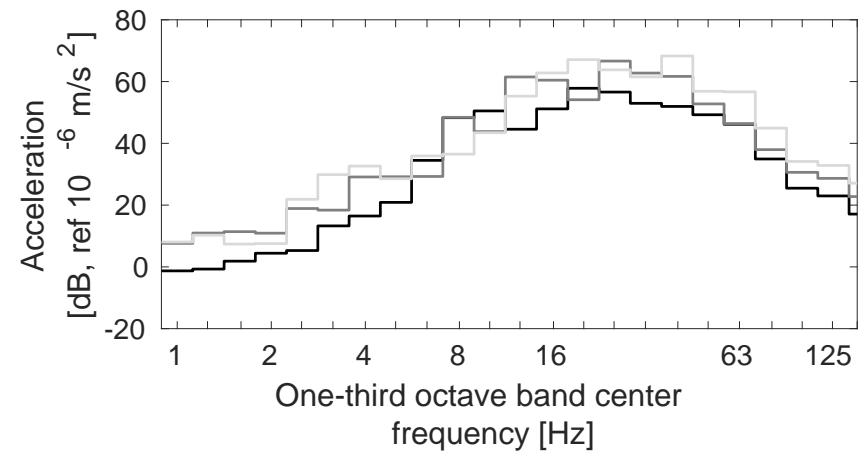

Figure 14. One-third octave band center frequency of the vertical weighted acceleration in the free-field due to a S-100 train passage at (black line) $v=100 \mathrm{~km} / \mathrm{h}$, (dark grey line) $v=150 \mathrm{~km} / \mathrm{h}$ and (light grey line) $v=200 \mathrm{~km} / \mathrm{h}$ at $20 \mathrm{~m}$. 
Finally, Figure 15 correlates train speed with both the MTVV of the free-field acceleration and the overall RMS value (Equation (21)) of the building response. A clear trend is observed, with vibration levels increasing with train speed. However, the amplification in the building response in relation to the soil response does not depend on the train speed.

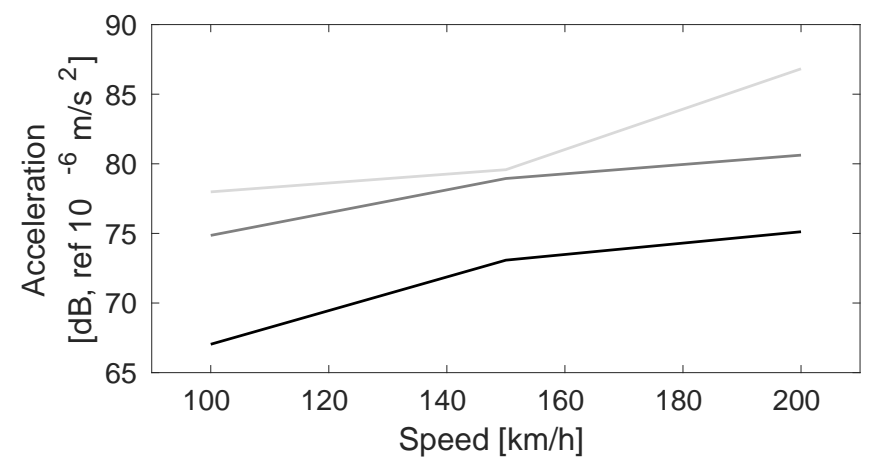

Figure 15. (Black line) MTVV at the free-field and overall RMS value of the weighted acceleration for the observation points (dark grey line) $A$ and (light grey line) $B$ at the top floor, due to a S-100 train passage at $20 \mathrm{~m}$ depending on the speed of train.

\section{4. $V_{s 30}$ parameter}

$V_{s 30}$ is a measure of the mean shear wave speed in the top $30 \mathrm{~m}$ of soil (Eurocode8 (1998)). It is a property commonly used in fields such as earthquake engineering as an estimate of surface shear wave velocity. Databases of $V_{s 30}$ values exist that cover the entire earth's landmass, meaning that $V_{s 30}$ can potentially be used to increase the accuracy of desktop vibration scoping studies. However, a challenge is that the mean shear wave velocity over a $30 \mathrm{~m}$ depth is typically greater than the shear wave speed at the uppermost soil surface (i.e. where ground-borne vibration is most efficient). Therefore, although a recognised parameter in international standards (Eurocode8 (1998); NEHRP (2015)), there have been studies about its limitations (L.A. Wald and J. Mori (2000); Castellaro et al. (2008); V.W. Lee and M.D. Triufnac (2010); L.A. Wald and J. Mori (2016)). Therefore the accuracy of using $V_{s 30}$ to approximate layered soils, within a railway vibration setting is investigated.

To do so, results for layered soils are compared with those obtained for a homogeneous soil considering $c_{s}=V_{s 30}$. Hereafter the homogeneous soil with $c_{s}=V_{s 30}$ is called equivalent homogeneous soil. Three layered soils and the equivalent homogeneous soils are considered (Table 6 ). The layered soil properties are chosen to ensure $V_{s 30}$ match the $c_{s}$ properties shown in Table 5 .

The twelve storey building (Figure 4) and the classical ballasted track on an embankment described previously in Section 2 (Table 1) are considered again.

Figure 16 shows the rail receptances for the layered soils (Table 6) and the equivalent homogeneous soils. It is observed that peaks in the track response for the three layered soils are found in the frequency 
Table 6. Layered soil properties.

\begin{tabular}{l|l|ccccc|c}
\hline & & $h[\mathrm{~m}]$ & $c_{p}[\mathrm{~m} / \mathrm{s}]$ & $c_{s}[\mathrm{~m} / \mathrm{s}]$ & $\xi[-]$ & $\rho\left[\mathrm{kg} / \mathrm{m}^{3}\right]$ & $V_{s 30}[\mathrm{~m} / \mathrm{s}]$ \\
\hline \multirow{3}{*}{ Soft } & Layer 1 & 24.1 & 318.9 & 159.5 & 0.05 & 1800 & \multirow{2}{*}{172.6} \\
& Half-space & $\infty$ & 518.1 & 259.1 & 0.05 & 1800 & \\
\hline \multirow{5}{*}{ Medium } & Layer 1 & 1.7 & 220.9 & 110.5 & 0.05 & 1800 & \\
& Layer 2 & 7.8 & 479.4 & 239.7 & 0.05 & 1800 & \multirow{3}{*}{334.9} \\
& Layer 3 & 2.7 & 726 & 363 & 0.05 & 1800 & \\
& Half-space & $\infty$ & 1038 & 519 & 0.05 & 1800 & \\
\hline \multirow{5}{*}{ Stiff } & Layer 1 & 2 & 361.5 & 180.7 & 0.05 & 1800 & \\
& Layer 2 & 3.6 & 660.4 & 330.2 & 0.05 & 1800 & \multirow{2}{*}{496.8} \\
& Layer 3 & 1.8 & 1113.2 & 556.6 & 0.05 & 1800 & \\
& Half-space & $\infty$ & 1291.6 & 645.8 & 0.05 & 1800 & \\
\hline
\end{tabular}

range $12 \mathrm{~Hz}$ to $16 \mathrm{~Hz}$. This is because the dominant frequency is strongly dependent upon the properties of the uppermost soil layer, which are similar for the three layered soils. A better agreement in terms of peak amplitudes is obtained at high frequencies. Regarding the three different soils, the homogeneous approximation performs best for the soft soil. This is because it has a smoother soil stratigraphy, characterised by a smaller discrepancy between the upper and lower layers' stiffness.

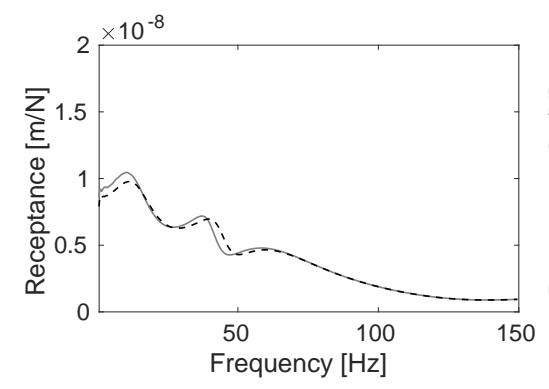

(a)

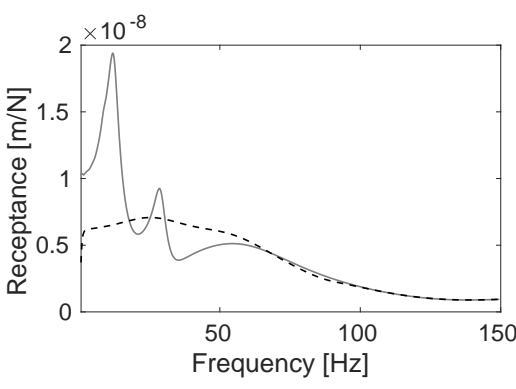

(b)



(c)

Figure 16. (Grey line) The displacement of the rail for the layered (a) soft, (b) medium and (c) stiff soils. (Black dashed line) Superimposed is the solution for the equivalent homogeneous soil .

Figure 17 shows the influence of soil stratigraphy on free-field mobility. In these results, it should be remembered that the neural network approach only utilises the upper layer properties $\left(h_{1}\right.$ and $\left.c_{s_{1}}\right)$ and the $V_{s 30}$ parameter (Figure 3 ), meaning the full soil profile is not considered. Regarding mobility results the level of error is similar to the receptance results, with the soft soil showing better agreement compared to the medium and stiff soils.

Figure 18 shows frequency contents and the running RMS values (ISO2631 (2003a)) of the free-field response, due to a S-100 train passage at $v=100 \mathrm{~km} / \mathrm{h}$. It is seen for the soft and stiff soils there are discrepancies at low frequency range, and for the medium and stiff soils at mid frequencies. Also, at some frequencies there are errors up to $13 \mathrm{~dB}$ in the frequency range $0 \mathrm{~Hz}$ to $100 \mathrm{~Hz}$. Regarding the running RMS curves, the correlation is very similar, with a maximum error of $9 \mathrm{~dB}$ for the stiff soil. 


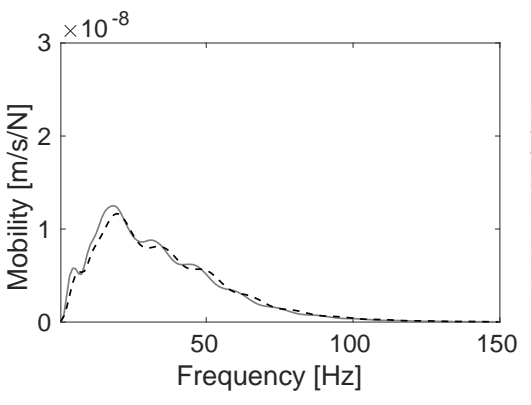

(a)

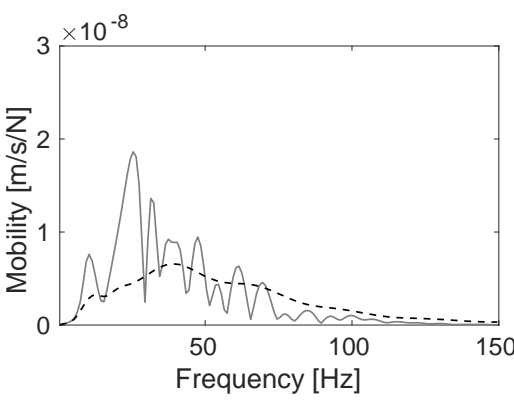

(b)

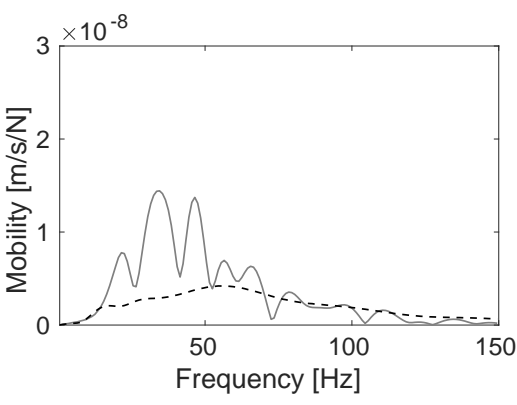

(c)

Figure 17. (Grey line) Free-field vertical mobility at $20 \mathrm{~m}$ from the axis track for the layered (a) soft, (b) medium and (c) stiff soils. (Black dashed line) Superimposed is the solution for the equivalent homogeneous soil.

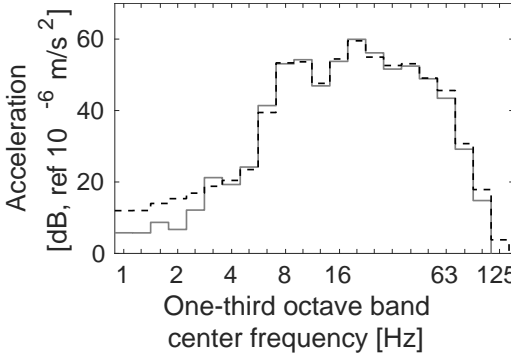

(a)

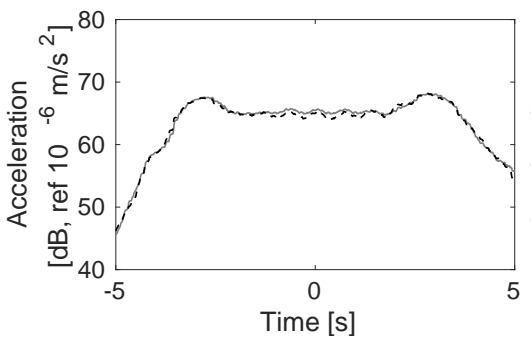

(d)



(b)

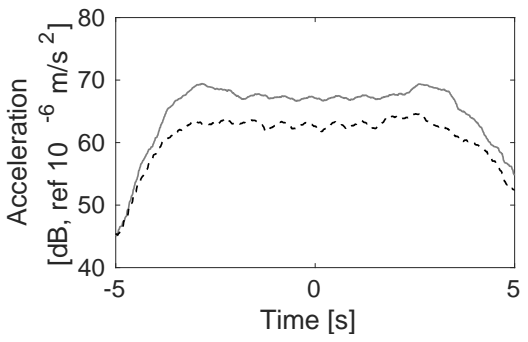

(e)

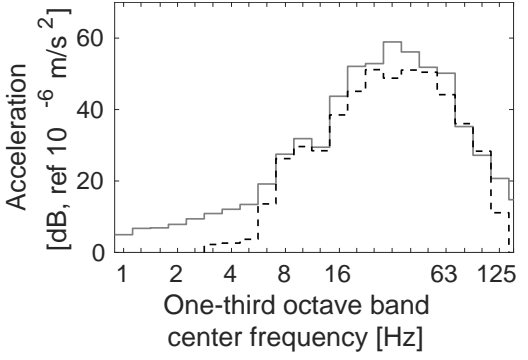

(c)

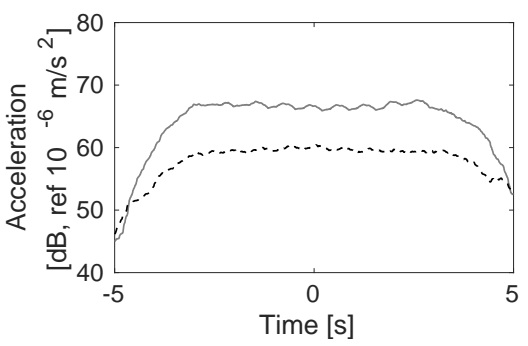

(f)

Figure 18. (Grey line) (a-c) One-third octave band center frequency and (d-f) running RMS value of the vertical weighted acceleration in the free-field at $20 \mathrm{~m}$ from the axis track due to a S-100 train passage at $v=100 \mathrm{~km} / \mathrm{h}$ for the layered (a and d) soft, (b and e) medium and ( $c$ and $f$ ) stiff soils. (Black dashed line) Superimposed is the solution for the equivalent homogeneous soil.

After the track and free-field responses, the building response is studied. Figure 19 shows that differences in ground-borne response spectra are in accordance with those observed previously in the free-field response (Figure 18), where results for the stiff soils present the highest discrepancies.

Figure 20 shows the contribution to the response of the building mode shapes. The small differences observed in the results for the soft soil (Figure 20 ( $a$ and d)) are mainly due to the foundation system of springs and dampers that represents the SSI (Equations (28) and (29)) which depends on the uppermost soil layer stiffness $c_{s 1}$. Taking into account $c_{s 1}<V_{s 30}$, this system has a lower energy dissipation for the 


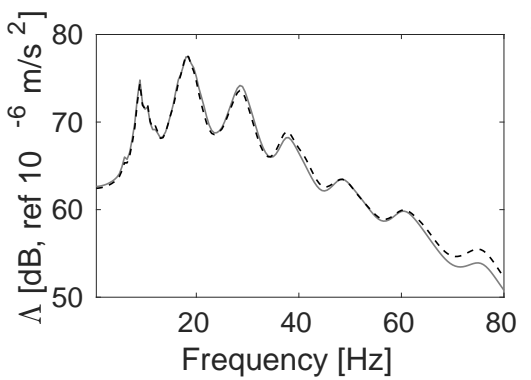

(a)

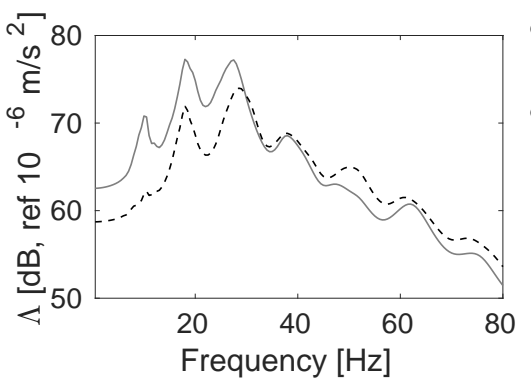

(b)

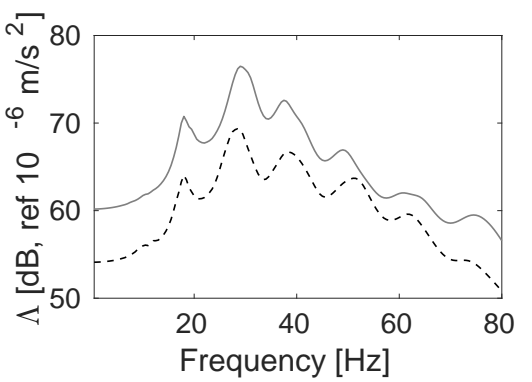

(c)

Figure 19. (Grey line) Ground-borne response spectra $\Lambda_{i}^{j}\left(f_{i}\right)$ at $20 \mathrm{~m}$ from the axis track due to a S-100 train passage at $v=100 \mathrm{~km} / \mathrm{h}$ for the layered (a) soft, (b) medium and (c) stiff soils. (black dashed line) Superimposed is the solution for the equivalent homogeneous soil.

equivalent homogeneous soil. Also, for the medium soil predictions, it is seen that modes of vibration are found at different frequencies (Figure 20 ( $b$ and e)) for both the layered and the equivalent homogeneous soils. Regarding the results for the stiff soil (Figure 20 (c and f)), the response of the equivalent homogeneous soil is underestimated at the dominant frequencies.

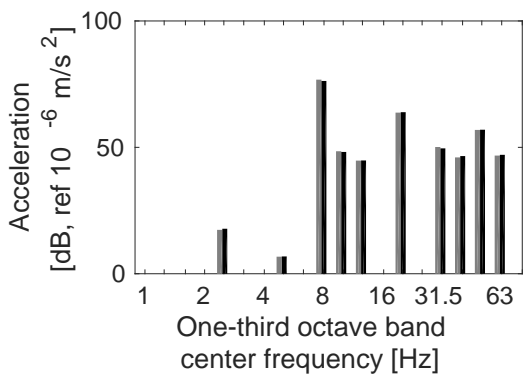

(a)

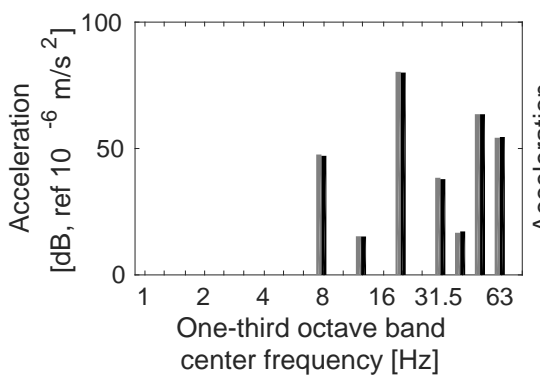

(d)

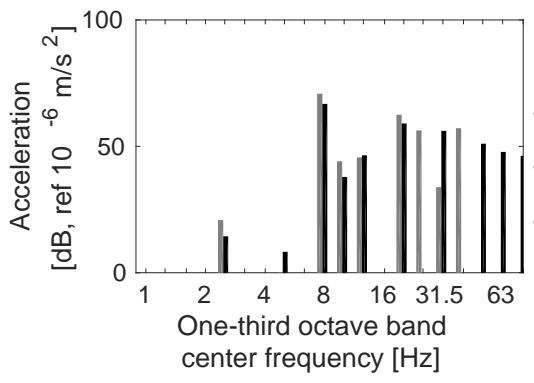

(b)

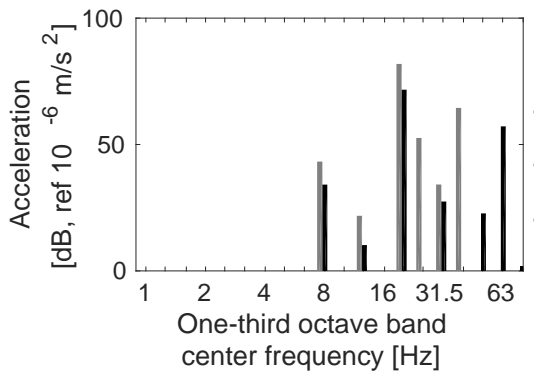

(e)

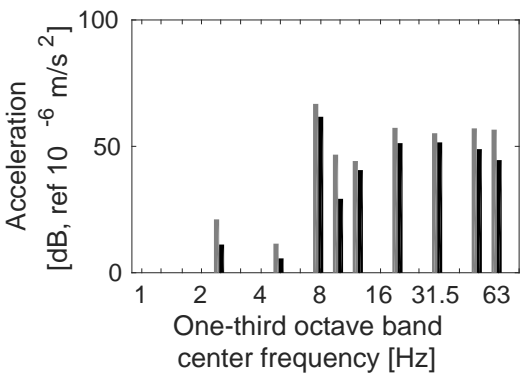

(c)

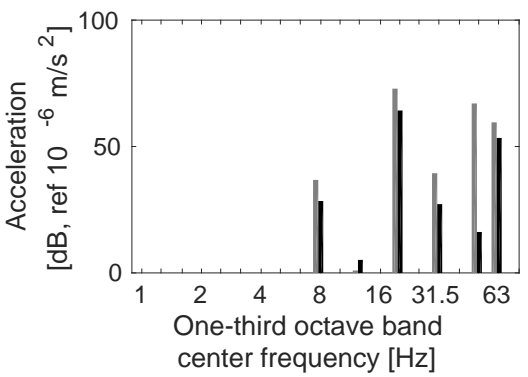

(f)

Figure 20. (Grey bars) Contribution of the modes to the overall RMS value of the vertical weighted acceleration of the building response at the top floor of the observation points ( $a, b$ and $c$ ) $A$ and ( $d$, e, and $\mathrm{f}$ ) $B$, for the layered (a and d) soft, ( $b$ and e) medium and ( $c$ and $f$ ) stiff soils. (Black bars) Superimposed is the solution for the equivalent homogeneous soil.

Figure 21 presents the overall RMS value (Equation (21)) of the free-field and twelve storey building vibration levels, considering different $V_{s 30}$ values. The soil response for homogeneous soils decreases 
as soil stiffness increases, however, the response of the layered soils show a contrasting behaviour. This is due to the shear wave velocity of the upper layer in each soil (Table 6). In all cases, this shear wave velocity is lower than the $V_{s 30}$ parameter and the difference between both increases from the soft to the stiff soil. This behaviour propagates to building response, where differences of approximately $10 \mathrm{~dB}$ are found. Predictions using the equivalent homogeneous soils underestimate the railway vibrations for the layered soils which is undesirable for the scoping model because it is designed to give conservative predictions. Therefore, it is concluded that the use of $V_{s 30}$ for ground vibration prediction should be used carefully and only for cases with straightforward stratigraphies.

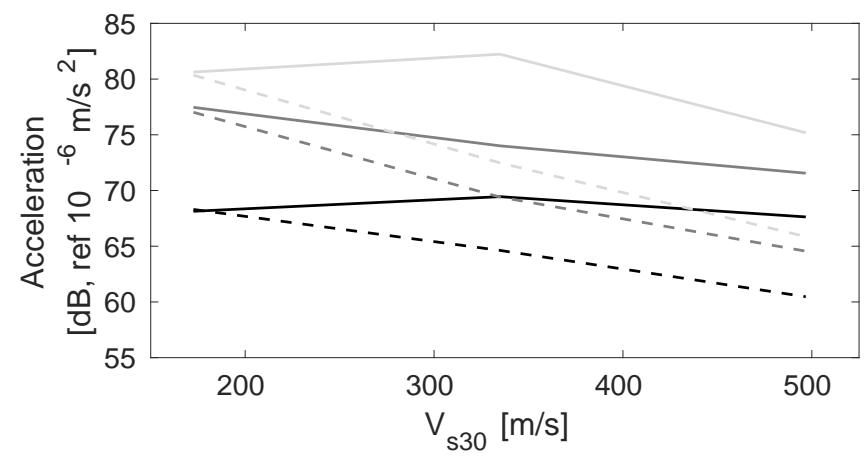

Figure 21. (Black line) MTVV at the free-field and overall RMS value of the weighted acceleration at the top floor of the observation points (dark grey line) $A$ and (light grey line) $B$, due to a $\mathrm{S}-100$ train passage at $v=100 \mathrm{~km} / \mathrm{h}$ considering (solid lines) layered and (dashed lines) homogeneous soils charecterized by their $V_{s 30}$ parameter.

\section{Discussion}

In an attempt to make a global uncertainty comparison, predictions from the scoping model and a more comprehensive 'reference' approach are presented. The reference model is developed using a BEM-FEM approach (Figure 22). Source and wave propagation mechanisms (Figure 22, step 2.3) are decoupled from the wave building immission (Figure 22, step 2.4). The reference model combined two methodologies. The first method is used to compute the source-propagation problem of waves in the soil and the second one to study the immission problem due to waves propagation in buildings:

- The first method (Figure 22, steps 2.1, 2.2 and 2.3) is based upon a 2.5D BEM-FEM methodology in the frequency-wavenumber domain (Lombaert et al. (2006); Galvín et al. (2010)). It is designed to compute the generation of railway vibrations and their propagation through the neighbouring soil. The train-track forces $\vec{g}(\omega)$ are calculated considering both quasi-static and dynamic contributions (Figure 22, step 2.1). Next, the track-soil transfer function $\tilde{\vec{u}}_{\mathrm{ff}}$ is calculated by modelling the track using FEM and the soil using BEM. The soil response is considered due to an impulse load applied on the rails (Figure 22, step 2.2). Train-track interaction forces are combined with the track-soil transfer function, resulting in the free-field response due to train passage $\vec{u}_{\mathrm{s}}$ at a point $\vec{x}$ (step 2.3). 


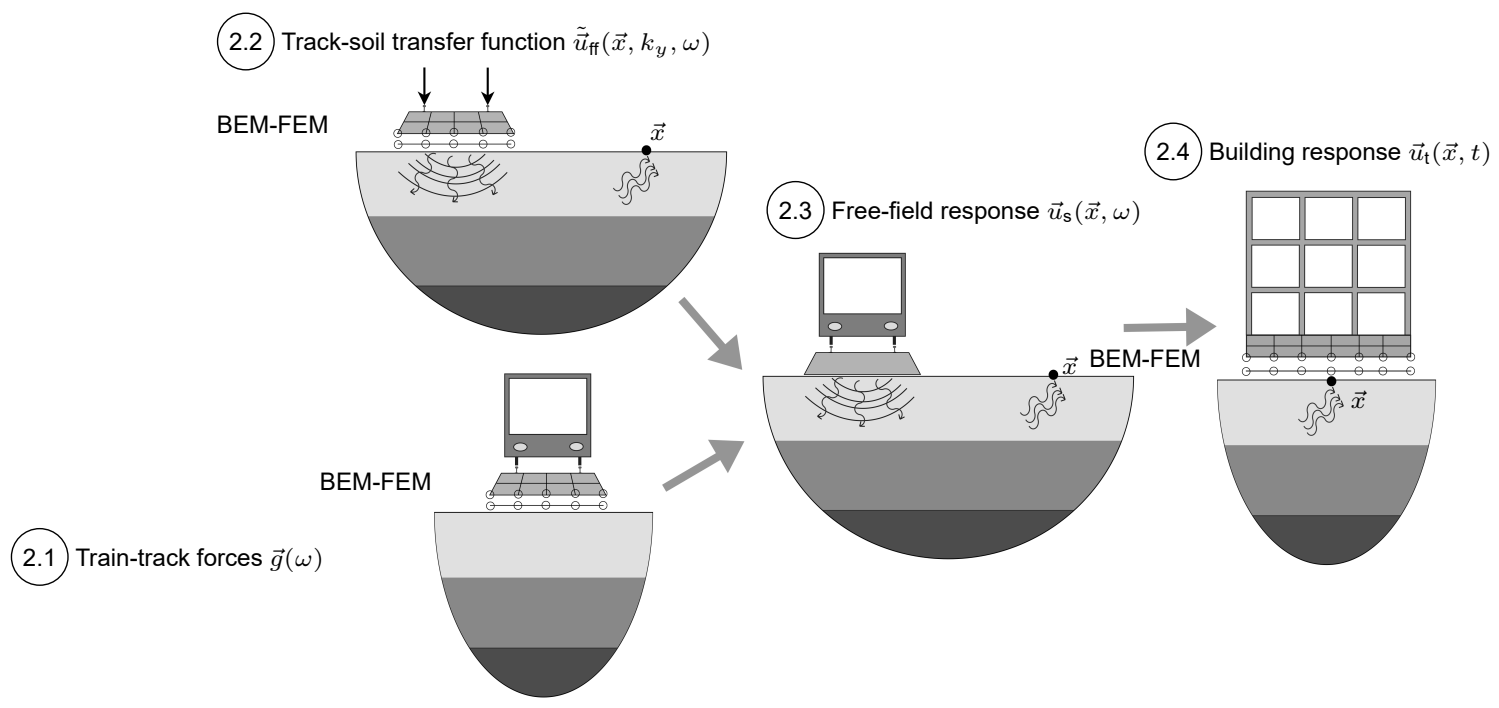

Figure 22. Scheme of the reference model.

- The second approach is used to obtain building vibrations due to a train passage. The foundation of the building is excited by the previously obtained free-field vibrations due to a train passage. The dynamic response of the building $\vec{u}_{\mathrm{t}}$ (Figure 22, step 2.4) is computed using the SSIFiBo toolbox based on a 3D time domain BEM-FEM methodology (Galvín and Romero (2014)).

The discrepancies between scoping and reference model results for all the cases previously presented in this study are analysed. Only free-field responses are included because the uncertainty of the scoping model to solve the immission problem of waves in buildings was studied enough previously by LópezMendoza et al. (2017). The authors found an uncertainty range between $-3 \mathrm{~dB}$ and $11 \mathrm{~dB}$ for building vibrations computed from the scoping model.

The difference in soil vibration predictions is calculated as $\Delta a=20 \log _{10}\left(\Lambda^{P} / \Lambda^{R}\right)$, where $\Lambda^{P}$ and $\Lambda^{R}$ are the the ground-borne response spectra from the scoping and the reference model, respectively. Ground-borne response spectra $\Lambda$ involves the building excitation in the scoping model (Equation (24)), represents a better way to determine the accuracy of the proposed model to solve the sourcepropagation problem of waves in the soil.

Figure 23 shows the discrepancy between both models for all the cases. Prediction accuracy is good, with $98.5 \%$ of the samples having an uncertainty in the range $-7.5 \mathrm{~dB}$ to $7.5 \mathrm{~dB}$.

The global uncertainty of the scoping model is determined using the MTVV vibration metric (ISO2631 (2003a)). Figure 24 presents the response for all the cases and a good agreement is found, with differences concentrated between $-4.8 \mathrm{~dB}$ to $5.6 \mathrm{~dB}$. Therefore the accuracy is similar to the uncertainty 


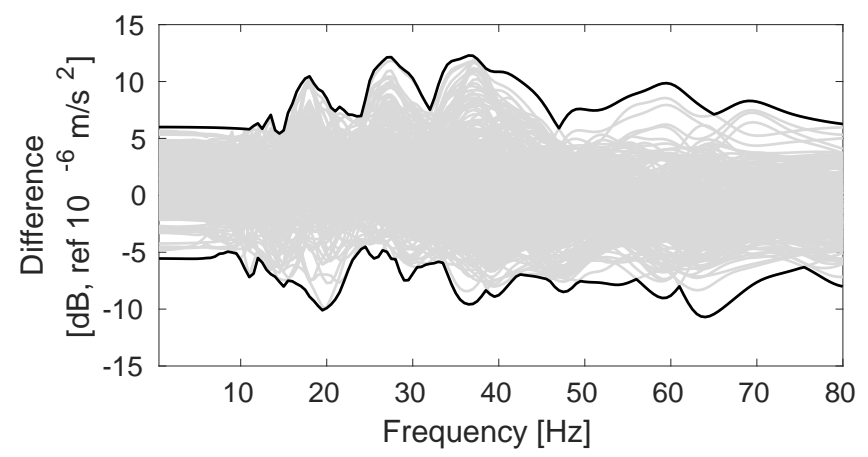

Figure 23. (Grey lines) Frequency content of the differences $\Delta a$ for all the cases. (Black lines) Superimposed is the envelope of the highest discrepancies.

range between $5 \mathrm{~dB}$ to $20 \mathrm{~dB}$ as found using more comprehensive models (Lombaert et al. (2014); Jones et al. (2012); Connolly et al. (2015b)).

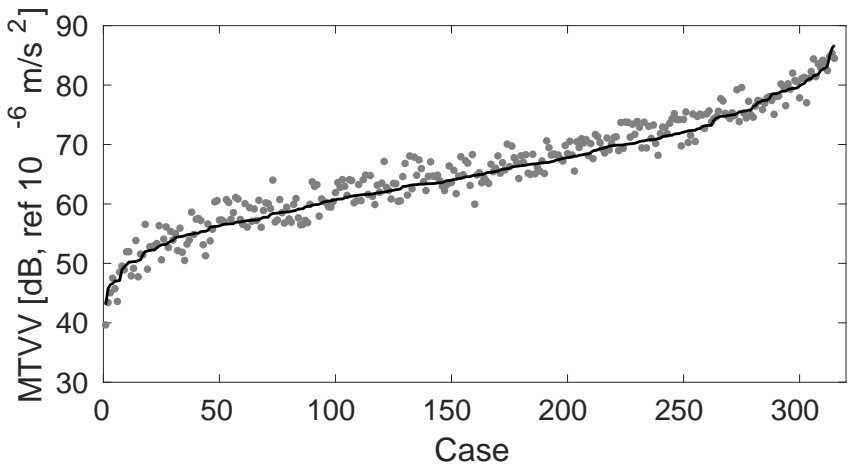

Figure 24. MTVV in the free-field due to a S-100 train passage for all the cases, computed by (black line) the reference model and (grey points) the scoping model.

An important advantage of the new scoping model compared to comprehensive models is its computational efficiency. Table 7 shows the computational costs to obtain the response of the twelve storey building due to a S-100 train travelling at $v=100 \mathrm{~km} / \mathrm{h}$ using an Intel One Core i7@1.87 GHz computer. The CPU time refers to the source-propagation problem of waves in the soil and the immission problem of waves in the building. Taking into account the architecture of the scoping and reference models (Figures 1 and 22), Table 7 outlines the main calculation steps and their run times. Note that the time required to obtain free-field predictions (step 2.3) is not presented because the models were identical. It should be noted that:

- The simplified track model (Figure 2) allows the scoping model to reduce computations of the prediction of train-track excitation $\vec{g}$ (step 2.1). The reference model uses a BEM-FEM methodology to calculate the train-track excitation, thus requiring additional computations. 
- The time required to calculate the track-soil transfer function $\tilde{\vec{u}}_{\mathrm{ff}}$ (step 2.2) using the scoping model is primarily due to the evaluation of the soil Green's function (step 2.2.2). The correction factor $\tilde{A}_{g}$ is estimated through a NN approach (step 2.2.1) and requires a minimal computational cost. The combination of these two steps results in a run time that is much lower than that for the reference model.

- The largest boost in computation efficiency come from the prediction of building response. Regarding the reference model computational cost, it should be noted that the cost required to compute the soil BEM domain under the building foundation is not included, and the CPU time is obtained from parallel computing.

The difference in run times is mainly due to the more comprehensive BEM-FEM methodology used in the reference model to consider track-soil and soil-structure interactions. Alternatively, the time required for the scoping to solve the whole vibration analysis from adding source-propagation and immission problems, is much lower than the necessary for the reference model.

Table 7. Average running time.

\begin{tabular}{c|cc}
\hline Step & Reference model & Scoping model \\
\hline Train-track forces & $25 \mathrm{~min}$ & $38 \mathrm{~s}$ \\
Track-soil transfer function & $15 \mathrm{~min}$ & $5 \mathrm{~min}$ \\
Building response & $37 \mathrm{~min}$ & $12 \mathrm{~s}$ \\
\hline
\end{tabular}

Considering these much reduced computational requirements, the range of uncertainty and the versatility of the proposed scoping model, it is concluded that it could be a powerful tool during the early design stages of railway lines where a large number of building vibrations assessment is required.

\section{Conclusions}

In this chapter, a simplified methodology to compute the propagation of railway vibrations into nearby buildings is presented. The model is novel because it is able to simulate the generation, propagation and immission of vibrations, for complex vehicle, track, soil and building arrangements in minimal time. The model is designed to be conservative in nature, to ensure that it is able to identify any high risk vibration sites during scoping tests, thus avoiding the application of any safety factor (e.g. $10 \mathrm{~dB}$ is commonly added in practise).

To do so, the source-propagation of wave energy through the ground has been decoupled from the immission of waves within buildings. Then, a simplified 2.5D FEM track model, a hybrid direct stiffnessneural network procedure and a modal superposition analysis for building response have been combined to create an overall model describing the vehicle-track-soil-building problem. 
A sensitivity analysis is undertaken using the scoping model. Track type, soil stiffness, building height and train speed effects are studied and it is found that there is a strong relationship between vibration levels and these parameters.

Comparisons have been made to determine the accuracy of using a global database of $V_{s 30}$ soil properties to predict vibration levels. Track receptance, free-field mobility, soil vibration, dominant building modes and building response due to railway traffic have been analysed and it has been found that this simplification is only satisfactory for cases with smooth stratigraphies.

Finally, results from the proposed model and a reference BEM-FEM approach have been compared and the new model has presented a good prediction ability. Discrepancies are due to modelling disparities between its architecture and that of the reference model.

In conclusion, the scoping model acts as a powerful MATLAB toolbox that allows users to evaluate freefield and building vibrations due to train passage at the early design stage.

\section{Acknowledgements}

This research was funded by the Spanish Ministry of Economy and Competitiveness (Ministerio de Economía y Competitividad) through research project BIA2016-75042-C2. Financial support is gratefully acknowledged. The support given by the Andalusian Scientific Computing Centre, the Leverhulme Trust and the University of Leeds Cheney Award Scheme is also gratefully acknowledged.

The first author would like to thank the Spanish Ministry of Education, Culture and Sport (Ministerio de Educación, Cultura y Deporte) for the financial support of his research stay at the School of Civil Engineering of the University of Leeds through the scholarship "Salvador de Madariaga" Reference PRX18/00115.

\section{References}

Auersch, L., 2005. The excitation of ground vibration by rail traffic: theory of vehicle-track-soil interaction and measurements on high-speed lines. Journal of Sound and Vibration 284, 103-132.

Auersch, L., 2010. Building Response due to Ground Vibration-Simple Prediction Model Based on Experience with Detailed Models and Measurements. International Journal of Acoustics and Vibration 15, 101-112.

Auersch, L., Romero, A., Galvín, P., 2014. Respuesta dinámica de edificaciones producida por campos de onda incidentes considerando la interacción suelo-estructura. Revista Internacional de Métodos Numéricos para Cálculo y Diseño en Ingeniería 30, 256-263.

Beale, M.H., M. T. Hagan, H. B. Demuth, 2017. Neural network toolbox User's guide. Mathworks, Inc.

Castellaro, S., Mulargia, F., P.L. Rossi, 2008. Vs30: Proxy for seismic amplification? Seismological Research Letters 79, 540-543.

Clough, R., Penzien, J., 1975. Dynamic of Structures. McGraw-Hill, New York. 
Connolly, D., G.P. Marecki, Kouroussis, G., Thalassinakis, I., P.K. Woodward, 2015a. The growth of railway ground vibration problems - A review. Science of the Total Environment 568, 1276-1282.

Connolly, D., Kouroussis, G., Giannopoulos, A., Verlinden, O., P.K. Woodward, M.C. Forde, 2014a. Assesment of railway vibrations using an efficient scoping model. Soil Dynamics and Earthquake Engineering 58, 37-47.

Connolly, D., Kouroussis, G., P.K. Woodward, Giannopoulos, A., Verlinden, O., M.C. Forde, 2014b. Scoping prediction of re-radiated ground-borne noise and vibration near high speed rails lines with variable soils. Soil Dynamics and Earthquake Engineering 66, 78-88.

Connolly, D., P. Alves Costa, Kouroussis, G., Galvín, P., P.K. Woodward, Laghrouche, O., 2015b. Large scale international testing of railway ground vibrations across Europe. Soil Dynamics and Earthquake Engineering 71, 1-12.

Cun, Y.L., Kanter, I., S. A. Solla, 1991. Second order properties of error surfaces: Learning time and generalization. Advances in Neural Information Processing Systems 3, 918-924.

DIN45672, 1995. DIN 45672 Teil 2: Schwingungsmessungen in der Umgebung von Schienenverkehrswegen: Auswerteverfahren. Deutsches Institut für Normung.

Eurocode8, 1998. Eurocode 8: Design of structures for earthquake resistance-Part 1 : General rules, seismic actions and rules for buildings. European Committee for Standardization.

François, S., Pyl, L., H.R. Masoumi, Degrande, G., 2007. The influence of dynamic soil-structure interaction on traffic induced vibrations in buildings. Soil Dynamics and Earthquake Engineering 27, 655-674.

François, S., Schevenels, M., Galvín, P., Lombaert, G., Degrande, G., 2010. A 2.5d coupled fe-be methodology for the dynamic interaction between longitudinally invariant structures and a layered halfspace. Computer Methods in Applied Mechanics and Engineering 199, 1536 - 1548.

Galvín, P., Domínguez, J., 2007. High-speed train-induced ground motion and interaction with structures. Journal of Sound and Vibration 307, 755-777.

Galvín, P., François, S., Schevenels, M., Bongini, E., Degrande, G., Lombaert, G., 2010. A 2.5d coupled fe-be model for the prediction of railway induced vibrations. Soil Dynamics and Earthquake Engineering 30, 1500 - 1512.

Galvín, P., Romero, A., 2014. A MATLAB toolbox for soil-structure interaction analysis with finite and boundary elements. Soil Dynamics and Earthquake Engineering 57, 10-14.

Galvín, P., Romero, A., Domínguez, J., 2010. Fully three-dimensional analysis of high-speed train-track-soil-structure dynamic interaction. Journal of Sound and Vibration 329, 5147-5163.

Galvín, P., Mendoza, D.L., Connolly, D., Degrande, G., Lombaert, G., Romero, A., 2018. Scoping assessment of free-field vibrations due to railway traffic. Soil Dynamics and Earthquake Engineering 114, 598-614.

Hagan, M.T., Menhaj, M.B., 1994. Training feedforward networks with the marquardt algorithm. EEE Transactions on Neural Networks 5, 989-993.

Hanson, C., D.A. Towers, L.D. Meister, 2005. High-speed ground Transportation Noise and Vibration Impact Assessment, HMMH Report 293630-4. U.S. Department of Transportation, Federal Railroad Administration, Office of Railroad Development .

Hanson, C., D.A. Towers, L.D. Meister, 2006. Transit Noise and Vibration Impact Assessment, Report FTA-VA-90-1003-06. U.S. Department of Transportation, Federal Transit Administration, Office of Planning and Environment .

Hussein, M., Hunt, H., Kuo, K., P. Alves Costa, Barbosa, J., 2013. The use of sub-modelling technique to calculate vibration in buildings from underground railways. Proceedings of the Institution of Mechanical Engineers, Part F: Journal of Rail and Rapid Transit 229, 303 - 314. 
ISO14837, 2005. ISO 14837-1:2005 Mechanical vibration-Ground-borne noise and vibration arising from rail systems-Part 1: General guidance. International Organization for Standardization.

ISO2631, 2003a. ISO 2631-1:2003: Mechanical vibration and shock-Evaluation of human exposure to whole-body vibration-Part 1: General requirements. International Organization for Standardization.

ISO2631, 2003b. ISO 2631-2:2003: Mechanical vibration and shock-Evaluation of human exposure to whole-body vibration-Part 2: Vibration in buildings $(1-80 \mathrm{~Hz})$. International Organization for Standardization.

Jean, P., Guigou, C., Villot, M., 2004. A 2.5D BEM Model for Ground-Structure Interaction. Building Acoustics 11, 1-17.

Jones, S., Kuo, K., M.F.M. Hussein, H.E.M Hunt, 2012. Prediction uncertainties and inaccuracies resulting from common assumptions in modelling vibration from underground railways. Proceedings of the Institution of Mechanical Engineers, Part F: Journal of Rail and Rapid Transit 226, 501-512.

Kausel, E., 2006. Fundamental solutions in elastodynamics: a compendium. Cambridge University Press, New York.

Kausel, E., Roësset, J.M., 1981. Stiffness matrices for layered soils. Bulletin of the Seismological Society of America 71, 1743.

Kouroussis, G., K.E. Vogiatzis, Connolly, D., 2017. A combined numerical/experimental prediction method for urban railway vibration. Soil Dynamics and Earthquake Engineering 97, 377 - 386.

Kuo, K., Verbraken, H., Degrande, G., Lombaert, G., 2016. Hybrid predictions of railway induced ground vibration using a combination of experimental measurements and numerical modelling. Journal of Sound and Vibration 373, $263-284$.

L.A. Wald, J. Mori, 2000. Evaluation of methods for estimating linear site-response amplifications in the los angeles region. Bulletin of the Seismological Society of America 90, S32-S42.

L.A. Wald, J. Mori, 2016. Comparisons between $V_{S 30}$ and spectral response for 30 sites in newcastle, australia, from collocated seismic cone penetrometer, active- and passive-source $V_{S}$ data. Bulletin of the Seismological Society of America 106, 1690-1709.

Lombaert, G., Degrande, G., 2009. Ground-borne vibration due to static and dynamic axle loads of intercity and high-speed trains. Journal of Sound and Vibration 319, 1036-1066.

Lombaert, G., Degrande, G., Kogut, J., François, S., 2006. The experimental validation of a numerical model for the prediction of railway induced vibrations. Journal of Sound and Vibration 297, 512 - 535.

Lombaert, G., Galvín, P., François, S., Degrande, G., 2014. Quantification of uncertainty in the prediction of railway induced ground vibration due to the use of statistical track unevenness data. Journal of Sound and Vibration 333, 4232 - 4253.

Lopes, P., P. Alves Costa, Ferraz, M., Calçada, R., A. Silva Cardoso, 2014. Numerical modeling of vibrations induced by railway traffic in tunnels: From the source to the nearby buildings. Soil Dynamics and Earthquake Engineering 61-62, 269-285.

López-Mendoza, D., Romero, A., Connolly, D., Galvín, P., 2017. Scoping assessment of building vibration induced by railway traffic. Soil Dynamics and Earthquake Engineering 93, 147-161.

Mezher, S., Connolly, D., P.K. Woodward, Laghrouche, O., Pombo, J., P.A. Costa, 2015. Railway critical velocity - analitical prediction and analysis. Transportation Geothechnics 2016, 84 - 96.

Monjezi, M., Ahmadi, M., Sheikhan, M., Bahrami, A., A. R. Salimi, 2010. Predicting blast-induced ground vibration using various types of neural networks. Soil Dynamics and Earthquake Engineering 30, 1233-1236.

NEHRP, 2015. NEHRP Recommended Seismic Provisions for New Buildings and Other Structures, FEMA P-1050. Building Seismic Safety Council.

Nourani, V., Fard, M.S., 2012. Sensitivity analysis of the artificial neural network outputs in simulation of the evaporation process at different climatologic regimes. Advances in Engineering Software 47, 127-146. 
P. Alves Costa, Calçada, R., A. Silva Cardoso, 2012. Track-ground vibrations induced by railway traffic: In-situ measurements and validation of a 2.5D FEM-BEM model. Soil Dynamics and Earthquake Engineering 32, $111-128$.

P. Alves Costa, Colaço, A., Calçada, R., A. Silva Cardoso, 2015. Critical speed of railway tracks. Detailed and simplified approaches. Transportation Geotechnics 2, 30-46.

Romero, A., Galvín, P., António, J., Domínguez, J., Tadeu, A., 2017. Modelling of acoustic and elastic wave propagation from underground structures using a 2.5d bem-fem approach. Engineering Analysis with Boundary Elements 76, 26 - 39.

Romero, A., Tadeu, A., Galvín, P., António, J., 2015. 2.5D coupled BEM-FEM used to model fluid and solid scattering wave. International Journal for Numerical Methods in Engineering 101, 148-164.

Rumelhart, D.E., McClelland, J.L., 1986. Parallel distributed processing: Explorations in the microstructure of cognition. Vol 1. MIT Press, Cambridge, MA.

Schevenels, M., François, S., Degrande, G., 2009. Edt: An elastodynamics toolbox for MATLAB. Computers \& Geosciences $35,1752-1754$.

Sheng, X., C.J.C. Jones, Thompson, D., 2006. Prediction of ground vibration from trains using the wavenumber finite and boundary element methods. Journal of Sound and Vibration 293, 575-586.

Triepaischajonsak, A., Thompson, D., 2015. A hybrid modelling approach for predicting ground vibration from trains. Journal of Sound and Vibration 335, $147-173$.

Verbraken, H., Lombaert, G., Degrande, G., 2011. Verification of an empirical prediction method for railway induced vibrations by means of numerical simulations. Journal of Sound and Vibration 330, 1692-1703.

V.W. Lee, M.D. Triufnac, 2010. Should average shear-wave velocity in the top $30 \mathrm{~m}$ of soil be used to describe seismic amplification? Soil Dynamics and Earthquake Engineering 30, 1250-1258.

Xia, H., Y.M. Cao, G. De Roeck, 2010. Theoretical modeling and characteristic analysis of moving-train induced ground vibrations. Journal of Sound and Vibration 329, 819-832.

Yurdakul, M., Akdas, H., 2013. Modeling uniaxial compressive strength of building stones using non-destructive test results as neural networks input parameters. Construction and Building Materials 47, 1010-1019. 